INT. J. CONTROL, 1991, VOL. 54, NO. 4, 763-791

\title{
Boundary control of a Timoshenko beam attached to a rigid body: planar motion
}

\author{
ÖMER MORGÜL $\dagger$
}

\begin{abstract}
A flexible spacecraft modelled as a rigid body which rotates in an inertial space is considered; a light flexible beam is clamped to the rigid body at one end and free at the other end. The equations of motion are obtained by using the geometrically exact beam model for the flexible beam, and it is then shown that under planar motion assumption, linearization of this model yields the Timoshenko beam model. It is shown that suitable boundary controls applied to the free end of the beam and a control torque applied to the rigid body stabilize the system. The proof is obtained by using a Lyapunov functional based on the energy of the system.
\end{abstract}

\section{Introduction}

Many mechanical systems, such as spacecraft with flexible appendages or robot arms with flexible links, can be modelled as coupled elastic and rigid parts. Many future space applications, such as the space station, rely on lightweight materials and high performance control systems for high precision pointing, tracking, etc., and to achieve high precision demands for such systems, one has to take the dynamic effect of flexible parts into account. Thus, over the last decade there has been a growing interest in obtaining new methods for the design, analysis and control of systems which have flexible parts. An excellent review of research in this area can be found in the work of Balas (1982).

The overall equations of motion of a system which has coupled elastic and rigid parts are usually a set of coupled non-linear ordinary and partial differential equations with appropriate boundary conditions. These equations can be obtained by using standard methods of mechanics (see for example Goldstein 1980). However, it should be noted that, although the mechanics of rigid bodies are well understood, this is not so for flexible bodies. There are many flexible body models and choosing one is not a trivial task (see e.g. Russell 1986).

In the analysis of such coupled rigid and flexible body systems, particularly in engineering applications the commonly used approach is to express the solutions (e.g. the displacements caused by elastic motion) as an infinite sum in terms of the eigenfunctions corresponding to the relevant (linear) partial differential equation, and then to consider only finitely many terms in this sum, see Meirovitch (1967), Balas (1982). This approach is called 'modal' analysis and reduces the original set of equations, which are coupled non-linear ordinary and partial differential equations, to a finite, although often very large, set of coupled non-linear ordinary differential equations. However, having established a control law for this reduced set of equations does not always guarantee that the same law will work on the original set of equations (e.g. one might encounter the so-called 'spillover'

Received 16 October 1989. Revised 25 December 1990

†Department of Electrical and Electronics Engineering, Bilkent University, 06533, Bilkent, Ankara, Turkey. 
problems - see Balas 1978). Also note that the actual number of modes of an elastic system, in theory, is infinite and the number of modes that should be retained is not known a priori.

The stability of systems which have elastic parts, particularly flexible space structures, has been studied in the past. Recently, Biswas (1986) used a Lyapunov type approach which uses the total energy of a flexible structure as a Lyapunov function to prove the stability of the system under appropriate control forces and torques applied to the flexible structure. The proposed control law contains distributed forces applied to the flexible structure (i.e. forces that are distributed over the flexible structure), which are proportional to the deflection velocities. Implementations of such control laws might not be easy or practical.

In recent years, the boundary control of flexible systems (i.e. controls applied to the boundaries of the flexible parts as opposed to controls distributed over the flexible parts), has become an important research area. Quinn and Russell (1978) established the uniform stabilization of the one-dimensional wave equation with boundary control, and later Chen (1979) obtained similar results for the wave equation in arbitrary space dimensions. More recently, Chen et al. (1987a, b) established the uniform stabilization of the Euler-Bernoulli beam with boundary control, and Kim and Renardy (1987) obtained similar results for the Timoshenko beam. In particular, Chen (1987 a) proved that in a cantilever beam, a single actuator applied at the free end of the beam is sufficient to stabilize the beam deflections uniformly and exponentially; and Delfour et al. (1986) investigated the case where the actuator is 'concentrated' on an area, as opposed to a single point. Recently, boundary control techniques have been applied to the stabilization of a flexible spacecraft, modelled as an Euler-Bernoulli beam attached to a rigid body, performing planar motion (Desoer and Morgül 1988), and three-dimensional motion (Morgül 1990).

In this paper, the motion of a flexible beam clamped to a rigid body at one end and free at the other end is studied. It is assumed that the mass of the rigid body is much larger than the mass of the flexible beam; hence it can be taken that the centre of mass of the rigid body is also the centre of mass of the whole system. It is also assumed that the centre of mass of the rigid body is fixed in an inertial frame. This assumption can be justified if one considers a satellite in a geosynchronous orbit, since in this case, neglecting the rotation of the Earth, the centre of mass of the satellite is fixed with respect to the Earth.

The so-called geometrically exact beam model for the flexible beam has been used (for details see Simo 1985, Vu-Quoc 1986). In $\S 2$, a basic review is given of this beam model. As an example, the equations of motion for the planar motion of a flexible beam modelled as a geometrically exact beam are obtained, and it is shown that linearization of the equations of motion for this particular case leads to the well-known Timoshenko beam equations (see Meirovitch 1967). Although the Timoshenko beam equations are more complex than those of the Euler-Bernoulli or Rayleigh beam equations, it is known that for large motions the results from the Timoshenko beam equations are in remarkably good agreement with those obtained from the exact theory of elasticity (see Bakr and Shabana 1987 and the references therein).

In $\S 3$ we introduce the basic rigid-body flexible-beam configuration studied in this paper, and using the geometrically exact beam model we obtain the equations of motion. We then define the rest state of this system and pose a stabilization 
problem: i.e., if the system is perturbed from the rest state, how may one find appropriate control laws that drive the system back to that rest state. We then propose a control law that consists the appropriate boundary control force and moments applied at the free end of the beam and a control torque applied to the rigid body.

In $\S 4$, it is first shown that the proposed control law stabilizes the system in the sense that the 'energy' of the system becomes a non-increasing function of time. Then, assuming planar motion, linearize the equations of motion of the beam, which results in the equations of motion for a Timoshenko beam, clamped to a rigid body at one end and free at the other end. We show that the proposed control law asymptotically stabilizes the system; more precisely, we prove that the 'energy' of this system decays as $O(1 / t)$ for large $t$.

In $\S 5$ we first give an existence and uniqueness result: then we prove that the 'energy' of the system decays exponentially to zero.

In $\S 6$, we present some simulation results and finally we give some concluding remarks.

\section{Review of the geometrically exact beam model}

\section{Kinematics}

Let the quadruople $\mathbf{N}=\left(\mathbf{e}_{1}, \mathbf{e}_{2}, \mathbf{e}_{3}\right)$ denote an inertial frame with the origin $\mathrm{O}$ and with the orthonormal dextral (i.e. right-handed) basis vectors $\mathbf{e}_{1}, \mathbf{e}_{2}, \mathbf{e}_{3}$. Let the flexible beam be initially straight along the $\mathbf{e}_{2}$ axis; we refer to this configuration as the reference configuration of the beam. We assume that one end of the beam is clamped to a rigid base fixed in $\mathbf{N}$ and the other end is free.

We associate the beam two geometrical objects: a set of planar cross-sections and a curve of centroids which pass through the centre of mass of each crosssection. Hence, in the reference configuration, the curve of centroids is a line along the direction of the $\mathbf{e}_{2}$ axis. Initially, we take the cross-sections perpendicular to this line. We assume that, throughout the motion, the planar sections remain planar. We also assume that the beam is homogeneous with uniform cross-sections.

Let $P$ be a typical beam element whose distance from $O$ in the reference configuation is $x$. Let $L$ be the length of the beam in the reference configuration, hence we have $0 \leqslant x \leqslant L$. We take $x$ as a coordinate along the curve of centroids. Let $\mathbf{r}(x, t)=\mathrm{OP}$ be the position vector of $\mathrm{P}$. Hence, initially we have $\mathbf{r}_{\mathrm{B}}(x, 0)=x \mathbf{e}_{2}$.

Let $C_{x}$ denote the cross-section at $x$ and let $\mathrm{P}$ also denote its centre at the curve of centroids. Let the quadruple $\left(P, d_{1}, d_{2}, d_{3}\right)$ denote a frame with its origin at $P$ and with orthonormal dextral vectors $d_{1}, d_{2}, d_{3}$ such that the axes $d_{1}, d_{3}$ lie in the cross-section $C_{x}$ and the axis $\mathrm{d}_{2}$ is normal to $C_{x}$ at all times. We denote this as the frame of directors at $x$.

Let $\Lambda(x, t)$ denote the orthogonal transformation matrix between the basis of the inertial frame $e_{1}, e_{2}, e_{3}$ and the basis of the frame of directors $d_{1}, d_{2}, d_{3}$; more precisely, we have for all $0 \leqslant x \leqslant L, t \geqslant 0$ :

$$
\mathbf{d}_{j}=\sum_{i=1}^{3} \Lambda_{i j}(x, t) \mathbf{e}_{i}, \quad j=1,2,3
$$

where $\Lambda_{i j}$ denotes the $(i, j)$ th component of the matrix $\Lambda$.

Initially choose

$$
\mathbf{d}_{j}(x, 0)=\mathbf{e}_{j}, \quad 0 \leqslant x \leqslant L \quad j=1,2,3
$$


Upon differentiating $\Lambda(x, t)$ with respect to $x$ and $t$ we obtain

$$
\frac{\partial}{\partial t} \Lambda(x, t)=W(x, t) \Lambda(x, t) \quad \frac{\partial}{\partial x} \Lambda(x, t)=\Omega(x, t) \Lambda(x, t)
$$

where, for all $t \geqslant 0,0 \leqslant x \leqslant L, W(x, t)$ and $\Omega(x, t)$ are $3 \times 3$ skew-symmetric matrices. Let the parameterization of $W$ and $\Omega$ be given by

$$
W:=\left[\begin{array}{ccc}
0 & -w_{3} & w_{2} \\
w_{3} & 0 & -w_{1} \\
-w_{2} & w_{1} & 0
\end{array}\right], \quad \Omega:=\left[\begin{array}{ccc}
0 & -\omega_{3} & \omega_{2} \\
\omega_{3} & 0 & -\omega_{1} \\
-\omega_{2} & \omega_{1} & 0
\end{array}\right]
$$

where, for the sake of simplicity, we suppress the dependency on $x$ and $t$.

We define the axial vectors $w$ and $\omega$ associated with the skew-symmetric matrices $W$ and $\Omega$ as follows:

$$
\mathbf{w}:=\sum_{i=1}^{3} w_{i} d_{i}, \quad \boldsymbol{\omega}:=\sum_{i=1}^{3} \omega_{i} d_{i}
$$

Following Simo (1985), we define the pull-back $\boldsymbol{\kappa}$ of $\omega$ by $\Lambda$, which is a measure of the curvature of the curve of centroids, and the strain measure $\Gamma$, as follows:

$$
\kappa:=\Lambda^{\mathrm{T}} \boldsymbol{\omega}, \quad \Gamma:=\Lambda^{\mathrm{T}} \frac{\partial \mathbf{r}}{\partial x}-\mathbf{e}_{2}
$$

where the superscript $T$ denotes the transpose.

We note the following relation between $\boldsymbol{\kappa}$ and $\omega$ (see Simo 1985):

$$
\frac{\partial \boldsymbol{\kappa}}{\partial t}=\Lambda^{\mathrm{T}} \frac{\partial \boldsymbol{\omega}}{\partial x}
$$

We define the contact force $\mathrm{n}(x, t)$ and the contact moment $\mathrm{m}(x, t)$ as follows. Consider a beam cross-section $C_{x}$ at $x$. The effect of the material which lies on the $(x, L)$ segment of the beam on the material which lies on the $[0, x]$ side of the beam is equivalent to a force and to a moment applied to the cross-section $C_{x}$, these are called the contact force and the contact moment, respectively (see Antman 1972).

\section{Dynamics}

We assume that the internal energy $\Psi$ of the beam is a function of $x, \boldsymbol{\kappa}$ and $\Gamma$ where the last two vectors are defined in (6). The equations of motion of a geometrically exact beam described above, together with the boundary conditions and the constitutive relations are given as follows, for all $0 \leqslant x \leqslant L, t \geqslant 0$ :

$$
\begin{gathered}
\frac{\partial \mathbf{n}}{\partial x}+\overline{\mathbf{n}}=\rho \frac{\partial^{2} \mathbf{r}}{\partial t^{2}} \\
\frac{\partial \mathbf{m}}{\partial x}+\frac{\partial \mathbf{r}}{\partial x} \times \mathbf{n}+\overline{\mathbf{1}}=I_{\mathbf{B}} \dot{\mathbf{W}}+\mathbf{W} \times I_{\mathbf{B}} \mathbf{W} \\
\mathbf{n}=\Lambda \frac{\partial \psi}{\partial \Gamma}, \quad \mathbf{m}=\Lambda \frac{\partial \psi}{\partial \mathbf{k}} \\
\mathbf{r}(0, t)=\mathbf{0}, \quad \Lambda(0, t)=I \\
\mathbf{n}(L, t)=\mathbf{f}(t), \quad \mathbf{m}(L, t)=\mathbf{g}(t)
\end{gathered}
$$


where $\overline{\mathbf{n}}$ and $\overline{\mathbf{m}}$ are the applied force and moment per unit length, respectively, $\rho$ is the mass per unit length of the beam, $I_{\mathrm{B}}$ is the inertia tensor of the beam cross-sections relative to the inertial frame $\mathbf{N}$, and $\mathbf{f}(t)$ and $\mathbf{g}(t)$ are the boundary control force and moment applied to the free end of the beam respectively.

Note that (8) is the balance of forces, (9) is the balance of moments at the beam cross-sections, (10) gives the constitutive relations, and (11) and (12) give the boundary conditions at the clamped and free ends, respectively.

\section{Special case: planar motion of a linear inextensible beam}

As an example, we consider the planar motion of the geometrically exact beam described above. We assume that the motion takes place in the plane spanned by the inertial axes $\mathbf{e}_{2}$ and $\mathbf{e}_{3}$ for all $t \geqslant 0$ and that we have $\boldsymbol{d}_{1}(x, t)=\mathbf{e}_{1}$, for all $0 \leqslant x \leqslant L, t \geqslant 0$. Let $\phi$ denote the angle between the axes $\boldsymbol{e}_{2}$ and $\mathrm{d}_{2}$, measured as the angle swept when the former axis is rotated around the axis $\mathbf{e}_{1}$ until its direction coincides with the direction of $d_{2}$. Hence, the rotation matrix $\Lambda$ takes the following form:

$$
\Lambda:=\left[\begin{array}{ccc}
1 & 0 & 0 \\
0 & \cos \phi & -\sin \phi \\
0 & \cos \phi & \cos \phi
\end{array}\right]
$$

therefore the axial vectors $\mathbf{w}, \boldsymbol{\omega}$ and the vector $\boldsymbol{\kappa}$ become

$$
\mathbf{w}=\frac{\partial \phi}{\partial t} \mathbf{d}_{1}, \quad \mathbf{\kappa}=\omega=\frac{\partial \phi}{\partial x} \mathbf{d}_{1}
$$

Let the position vector $\mathrm{r}(x, t)=\mathrm{OP}$ be parametrized as follows:

$$
\mathbf{r}(x, t):=x \mathbf{e}_{2}+u(x, t) \mathbf{e}_{3}
$$

where $u(x, t)$ denotes the beam displacement along the axis $\mathbf{e}_{3}$. Here we assume that the beam displacement along the axis $\mathbf{e}_{2}$ is identically zero, i.e. the beam is inextensible.

The contact force $\mathbf{n}$, the contact moment $\boldsymbol{m}$ and the strain measure $\boldsymbol{\Gamma}$ take the following form:

$$
\mathbf{n}=n_{2} \mathbf{e}_{2}+n_{3} \mathbf{e}_{3}, \quad \mathbf{m}=m_{1} \mathbf{e}_{1}, \quad \Gamma=\Gamma_{2} \mathbf{e}_{2}+\Gamma_{3} \mathbf{e}_{3}
$$

Using (6), assuming that $u$ and $\phi$ are small and neglecting higher order terms, we obtain

$$
\Gamma_{2}=0, \quad \Gamma_{3}=\frac{\partial u}{\partial x}-\phi
$$

As is often assumed in practice, we take the following quadratic form for the internal energy:

$$
\Psi=\frac{1}{2} \mathrm{GA} \Gamma_{3}^{2}+\frac{1}{2} \mathrm{EI} \kappa^{2}
$$

where $\kappa=\partial \phi / \partial x ; \mathrm{GA}$ is called the shear stiffness (along the axis $\mathrm{d}_{3}$ ); and EI is called the principal bending stiffness (relative to the axis $d_{1}$ ) (see Meirovitch 1967). 
Using (18) in (10), and then in (8), (9), and neglecting the axial motion, $\overline{\mathbf{n}}, \overline{\mathbf{m}}$, the equations of motion (8), (9) and the boundary conditions (11), (12) now become, for all $t \geqslant 0,0<x<L$ :

$$
\begin{gathered}
\mathrm{GA}\left(\frac{\partial^{2} u}{\partial x^{2}}-\frac{\partial \phi}{\partial x}\right)=\rho \frac{\partial^{2} u}{\partial t^{2}} \\
\text { EI } \frac{\partial^{2} \phi}{\partial x^{2}}+\mathrm{GA}\left(\frac{\partial u}{\partial x}-\phi\right)=I_{\mathrm{B}} \frac{\partial^{2} \phi}{\partial t^{2}} \\
u(0, t)=0, \quad \phi(0, t)=0 \\
\mathrm{GA}\left(u_{\mathrm{x}}(L, t)-\phi(L, t)\right)=f(t), \quad \text { EI } \phi_{\mathrm{x}}(L, t)=g(t)
\end{gathered}
$$

where we take $\mathbf{f}(t)=f(t) \boldsymbol{e}_{3}, \mathbf{g}(t)=g(t) \mathbf{e}_{1}$; also note that a variable sub-index denotes partial differentiation with respect to the variable, e.g. $u_{\mathrm{x}}=\partial u / \partial x$, etc.

We note that (19) and (20) are the equations of motion for a Timoshenko beam (see Meirovitch 1967).

\section{Equations of motion of flexible spacecraft}

Consider the following configuration: Fig. 1 shows the rigid body (drawn as a square) and the beam; $P$ is a point on the beam:

In Fig. 1 , the quadruple $\left(\mathrm{O}, \mathbf{e}_{1}, \mathbf{e}_{2}, \mathbf{e}_{3}\right)$ denotes a dextral orthonormal inertial frame, which will be referred to as $\mathbf{N}$, the quadruple $\left(O, D_{1}, D_{2}, D_{3}\right)$ denotes a dextral orthonormal frame fixed in the rigid body, which will be referred as $\mathbf{B}$, where $O$ is also the centre of mass of the rigid body and $D_{1}, D_{2}, D_{3}$ are along the principle axes of inertia of the rigid body. The beam is clamped to the rigid body at the point $Q$ at one end along the $D_{2}$ axis and is free at the other end. Let $L$ be the length of the beam. We assume that the mass of the rigid body is much larger than the mass of the beam, so that the centre of mass of the rigid body is approximately the centre of mass of the whole configuration. Hence, we assume that the point $O$ is fixed in the inertial space throughout the motion of the whole configuration. We also assume that the beam is inextensible (i.e. no deformation along the axis $\mathbf{D}_{2}$ ), and homogeneous with uniform cross-section.

The beam is initially straight along the $D_{2}$ axis. Let $P$ be a point on the curve of centroids whose distance from $\mathrm{Q}$ in the undeformed configuration is $x$ (i.e. when

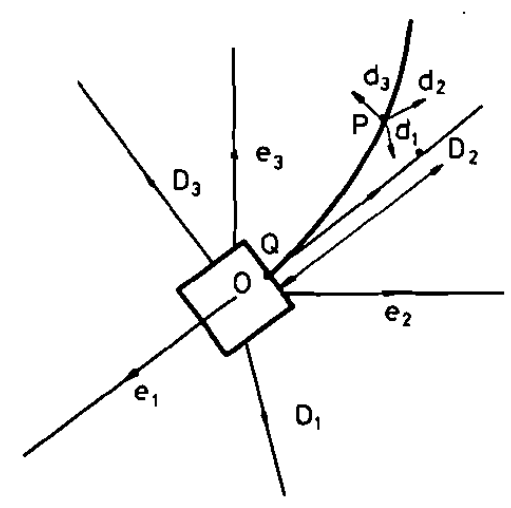

Figure 1. Rigid body with a flexible beam. 
the beam is straight along the $D_{2}$ axis), let the quadruple $\left(P, d_{1}, d_{2}, d_{3}\right)$ denote the frame of the directors located at $P$, where $d_{1}, d_{2}, d_{3}$ are the directors at $x$. Let $\mathbf{r}(x, t)=\mathrm{OP}$ be the position vector of $\mathrm{P}$.

Let, $\Lambda(x, t)$ be the orthogonal transformation between the body frame and the frame of directors. Then, neglecting gravitation and surface loads and assuming that the centre of mass of the rigid body is fixed in the inertial frame $\mathbf{N}$, the equations for flexible spacecraft are given by, for all $t \geqslant 0,0<x<L$ :

$$
\begin{gathered}
\frac{\partial \mathbf{n}}{\partial x}=\rho\left(\frac{\partial^{2} \mathbf{r}}{\partial t^{2}}\right)_{\mathrm{N}} \\
\frac{\partial \mathbf{m}}{\partial x}+\frac{\partial \mathbf{r}}{\partial x} \times \mathbf{n}=I_{\mathrm{B}}\left(\frac{\partial\left(\mathbf{w}+\boldsymbol{\omega}_{\mathrm{R}}\right)}{\partial t}\right)_{\mathrm{B}}+\left(\mathbf{w}+\boldsymbol{\omega}_{\mathrm{R}}\right) \times I_{\mathrm{B}}\left(\mathbf{w}+\boldsymbol{\omega}_{\mathrm{R}}\right) \\
I_{\mathrm{R}} \dot{\boldsymbol{\omega}}_{\mathrm{R}}+\omega_{\mathrm{R}} \times I_{\mathrm{R}} \omega_{\mathrm{R}}=\mathbf{r}(0, t) \times \mathbf{n}(0, t)+\mathbf{m}(0, t)+\mathbf{N}_{\mathrm{c}}(t) \\
\mathbf{n}=\Lambda \frac{\partial \psi}{\partial \Gamma}, \quad \mathbf{m}=\Lambda \frac{\partial \psi}{\partial \mathbf{K}} \\
\mathbf{r}(0, t)=\mathbf{O O}, \quad \Lambda(0, t)=I \\
\mathbf{n}(L, t)=\mathbf{f}(t), \quad \mathbf{m}(L, t)=\mathbf{g}(t)
\end{gathered}
$$

where $\mathrm{n}(x, t)$ and $\mathbf{m}(x, t)$ are the contact force and the contact moment of the beam, respectively; $(\partial(\cdot) / \partial t)_{N}$ denotes that the time differentiation is taken in the frame $N ; \rho$ is the mass per unit length of the beam; $I_{\mathrm{B}}$ is the inertia tensor of beam cross-sections, which is a constant matrix by assumption; $\omega_{\mathrm{R}}$ is the angular velocity of the rigid body in the inertial frame $\mathbf{N}$; $I_{\mathrm{R}}$ is the inertia tensor of the rigid body, which is a constant diagonal matrix by assumption; $\mathbf{N}_{\mathrm{c}}(t)$ is the control torque applied to the rigid body; $\Psi(\Gamma, \kappa)$ is the internal energy (i.e. potential energy) per unit length of the beam, which at the moment need not be a quadratic function of its arguments; and $\mathbf{f}(t)$ and $\mathbf{g}(t)$ are the boundary control force and moment, respectively, both applied at the free end of the beam. Also note that the time derivation between different frames are related as follows (see Goldstein 1980):

$$
\left(r_{t}\right)_{N}=\left(r_{t}\right)_{B}+\omega_{R} \times r
$$

Equations (23) and (24) state the balance of forces and the balance of moments at the beam cross-sections, respectively; $(25)$ is the rigid body angular momentum equation; (26) is the constitutive equation of the beam; and (27) and (28) give the boundary conditions at the clamped end and at the free end, respectively (for details, see Morgül 1989).

We define the rest state of the system given by $(23)-(28)$ as follows:

$$
\begin{gathered}
\omega_{\mathbf{R}}=\mathbf{0} \\
\frac{\partial \mathbf{r}}{\partial x}=\mathbf{D}_{2}, \quad x \in[0, L] \\
\Lambda(x)=I, \quad x \in[0, L]
\end{gathered}
$$

It is easy to see that (30) holds for all $t \in \mathbb{R}_{+}$if and only if the rigid body does not spin in the inertial frame $\mathbf{N}$.

Let the curve of centroids be represented by

$$
\mathbf{r}(x, t)=u_{1} \mathbf{D}_{1}+\left(|\mathrm{OQ}|+x+u_{2}\right) \mathbf{D}_{2}+u_{3} \mathbf{D}_{3}
$$


and, by (27), $u_{1}(0, t)=u_{2}(0, t)=u_{3}(0, t)=0$ for all $t \geqslant 0$. Then (31) holds for all $t \in \mathbb{B}_{+}$if and only if the beam displacements $u_{1}, u_{2}, u_{3}$ are identically zero.

If (31) holds for all $t \in \mathbb{R}_{+}$, then the beam deflections $u_{1}, u_{2}, u_{3}$ do not depend on time, hence by the first boundary condition in (27) they are identically zero on $[0, L] \times \mathbb{R}_{+}$. Conversely, if $u_{1}, u_{2}, u_{3}$ are identically zero on $[0, L] \times \mathbb{R}_{+}$, then (31) trivially follows from (33).

Also note that (32) holds if and only if the strain measure $\kappa$ defined in (7) is identically zero on $[0, L] \times \mathbb{R}_{+}$. If (31) holds, then the second equation in (3) implies that the skew-symmetric matrix $\Omega(x, t)$ is identically zero on $[0, L] \times \mathbb{R}_{+}$, which then implies that the corresponding axial vector $\omega$ and hence the strain measure $\boldsymbol{\kappa}$ are all identically zero on $[0, L] \times \mathbb{R}_{+}$. Conversely, if $\boldsymbol{\kappa}$ is identically zero on $[0, L] \times \mathbb{R}_{+}$, then so is the axial vector $\omega$ and the corresponding skew-symmetric matrix $\Omega$. Then (3) implies that $\Lambda$ does not depend on $x$; hence by using boundary condition (5), we obtain (32). Furthermore, if (31) holds, (32) implies that the other strain measure $\Gamma$ defined in (6) is also identically zero on $[0, L] \times \mathbb{R}_{+}$.

\section{Stabilization problem}

The stabilization problem is stated as follows: let the system given by (23) $-(28)$ be disturbed from the rest state given by $(30)-(32)$; find appropriate control laws $\mathbf{N}_{\mathrm{c}}(t), \mathbf{f}(t), \mathbf{g}(t)$ that drive the system back to the rest state.

\section{Natural control law}

This control law applies a force $\mathrm{n}(L, t)$ and a torque $\mathrm{m}(L, t)$ at the free end of the beam and a torque $\mathbf{N}_{\mathrm{c}}(t)$ to the rigid body. They are specified as follows: choose $3 \times 3$ symmetric positive definite matrices $\mathbf{K}, \mathbf{L}, \mathbf{M}$ (which can all be chosen diagonal); then for all $t \geqslant 0$ the 'natural control law' requires that

$$
\begin{gathered}
\mathbf{n}(L, t)=-\mathbf{L}\left(\mathbf{r}_{\mathbf{t}}(L, t)\right)_{\mathbf{B}} \\
\mathbf{m}(L, t)=-\mathbf{M w}(L, t) \\
\mathbf{N}_{\mathrm{c}}(t)=-\mathbf{r}(L, t) \times \mathbf{n}(L, t)-\mathbf{m}(L, t)-\mathbf{K} \boldsymbol{\omega}_{\mathbf{R}}
\end{gathered}
$$

This control law is 'natural' in the sense that it enables us to use the total energy of the system as a Lyapunov function to study its stability.

\section{Stability results for the natural control law}

Consider the system given by (23) -(26) together with the control law (34)(36). To study the stability of this system, we define the energy of the system as follows for all $t \geqslant 0$ :

$$
\begin{aligned}
E(t)= & \frac{1}{2}\left\langle\boldsymbol{\omega}_{\mathrm{R}}, I_{\mathrm{R}} \omega_{\mathrm{R}}\right\rangle+\frac{1}{2} \int_{0}^{L} \rho\left\langle\mathbf{r}_{t}, \mathbf{r}_{t}\right\rangle d x \\
& +\frac{1}{2} \int_{0}^{L}\left\langle\left(\omega_{\mathrm{R}}+\mathbf{w}\right), I_{\mathrm{B}}\left(\omega_{\mathrm{R}}+\mathbf{w}\right)\right\rangle d x+\frac{1}{2} \int_{0}^{L} \Psi(\boldsymbol{\Gamma}, \boldsymbol{\kappa}) d x
\end{aligned}
$$

where $\langle\cdot, \cdot\rangle$ denotes the standard inner-product in $\mathbb{B}^{3}$, and $\mathbf{r}_{\boldsymbol{r}}$ is the abbreviation for $\left(r_{t}(x, t)\right)_{\mathrm{N}}$. The first term in (37) represents the rotational kinetic energy of the rigid body, the second term represents the kinetic energy of the beam in the inertial 
frame $\mathbf{N}$, the third term represents the rotational kinetic energy of the beam cross-sections and the last term represents the potential energy of the beam.

\section{Proposition}

Consider the system given by (23) $-(28)$ and (34) $-(36)$, which will be called the system $\mathscr{S}$. Then the energy $E(t)$ defined in (37) is a non-increasing function of time along the solutions of the system $\mathscr{S}$.

Proof

Differentiating (37) with respect to time $t$, we obtain

$$
\begin{aligned}
\frac{d E}{d t} & =\left\langle\boldsymbol{\omega}_{\mathrm{R}}, \frac{d}{d t}\left(I_{\mathrm{R}} \boldsymbol{\omega}_{\mathrm{R}}\right)\right\rangle+\int_{0}^{L} \rho\left\langle\mathbf{r}_{t}, \mathbf{r}_{t t}\right\rangle d x+\int_{0}^{L}\left\langle\left(\omega_{\mathrm{R}}+\mathbf{w}\right), \frac{d}{d t}\left[I_{\mathrm{B}}\left(\boldsymbol{\omega}_{\mathrm{R}}+\mathbf{w}\right)\right]\right\rangle d x \\
& +\int_{0}^{L}\left\langle\frac{\partial \Psi}{\partial \Gamma}, \frac{\partial \Gamma}{\partial t}\right\rangle d x+\int_{0}^{L}\left\langle\frac{\partial \Psi}{\partial \mathbf{K}}, \frac{\partial \mathbf{K}}{\partial t}\right\rangle d x \\
= & \left\langle\boldsymbol{\omega}_{\mathrm{R}}, I_{\mathrm{R}} \dot{\omega}_{\mathrm{R}}+\omega_{\mathrm{R}} \times I_{\mathrm{R}} \omega_{\mathrm{R}}\right\rangle+\int_{0}^{L}\left\langle\left(\mathbf{r}_{t}\right)_{\mathrm{B}}+\boldsymbol{\omega}_{\mathrm{R}} \times \mathbf{r}, \mathbf{n}_{\mathbf{x}}\right\rangle d x \\
& +\int_{0}^{L}\left\langle\left(\omega_{\mathrm{R}}+\mathbf{w}\right), I_{\mathrm{B}} \frac{\partial}{\partial t}\left(\omega_{\mathrm{R}}+\mathbf{w}\right)_{\mathrm{B}}+\left(\omega_{\mathrm{R}}+\mathbf{w}\right) \times I_{\mathrm{B}}\left(\omega_{\mathrm{R}}+\mathbf{w}\right)\right\rangle d x \\
& +\int_{0}^{L}\left\langle\frac{\partial \Psi}{\partial \Gamma}, \frac{\partial \Gamma}{\partial t}\right\rangle d x+\int_{0}^{L}\left\langle\frac{\partial \Psi}{\partial \mathbf{K}}, \frac{\partial \mathbf{k}}{\partial t}\right\rangle d x
\end{aligned}
$$

where in the second equation we use (23) and (29).

Using integration by parts, we calculate various integrals in (38) as follows:

$$
\begin{aligned}
\int_{0}^{L}\left\langle\left(\mathbf{r}_{t}\right)_{\mathbf{B}}, \mathbf{n}_{x}\right\rangle d x= & \left\langle\left(\mathbf{r}_{t}\right)_{\mathbf{B}}(L, t), \mathbf{n}(L, t)\right\rangle-\int_{0}^{L}\left\langle\left(\mathbf{r}_{t}\right)_{\mathbf{B}}, \mathbf{n}\right\rangle d x \\
\int_{0}^{L}\left\langle\boldsymbol{\omega}_{\mathbf{R}} \times \mathbf{r}, \mathbf{n}_{\mathbf{x}}\right\rangle d x= & \left\langle\boldsymbol{\omega}_{\mathbf{R}}, \int_{0}^{L} \mathbf{r} \times \mathbf{n}_{x} d x\right\rangle=\left\langle\boldsymbol{\omega}_{\mathrm{R}}, \mathbf{r}(L, t) \times \mathbf{n}(L, t)\right\rangle \\
& \quad\left\langle\boldsymbol{\omega}_{\mathbf{R}}, \int_{0}^{L} \mathbf{r}_{x} \times \mathbf{n} d x\right\rangle \\
I= & \left\langle\left(\boldsymbol{\omega}_{\mathrm{R}}+\mathbf{w}\right), I_{\mathbf{B}} \frac{\partial}{\partial t}\left(\boldsymbol{\omega}_{\mathbf{R}}+\mathbf{w}\right)_{\mathbf{B}}+\left(\boldsymbol{\omega}_{\mathbf{R}}+\mathbf{w}\right) \times I_{\mathbf{B}}\left(\boldsymbol{\omega}_{\mathrm{R}}+\mathbf{w}\right)\right\rangle d x \\
= & \left.\int_{0}^{L}\left\langle\boldsymbol{\omega}_{\mathbf{R}}+\mathbf{w}\right), \mathbf{m}_{x}+\mathbf{r}_{x} \times \mathbf{n}\right\rangle d x \quad \text { by }(24) \\
= & \int_{0}^{L}\left\langle\mathbf{w}, \mathbf{m}_{x}\right\rangle d x+\left\langle\boldsymbol{\omega}_{\mathbf{R}}, \int_{0}^{L} \mathbf{m}_{x} d x\right\rangle \\
& +\int_{0}^{L}\left\langle\mathbf{w}, \mathbf{r}_{x} \times \mathbf{n}\right\rangle d x+\left\langle\boldsymbol{\omega}_{\mathrm{R}}, \int_{0}^{L} \mathbf{r}_{x} \times \mathbf{n} d x\right\rangle \\
= & \langle\mathbf{w}(L, t), \mathbf{m}(L, t)\rangle-\left\langle\mathbf{w}(0, t), \mathbf{m}^{L}(0, t)\right\rangle \\
& -\int_{0}^{L}\langle\mathbf{w} x, \mathbf{m}\rangle d x+\left\langle\boldsymbol{\omega}_{\mathrm{R}}, \mathbf{m}(L, t)-\mathbf{m}(0, t)\right\rangle \\
& +\int_{0}^{L}\left\langle\mathbf{w} \times \mathbf{r}_{x}, \mathbf{n}\right\rangle d x+\left\langle\boldsymbol{\omega}_{\mathrm{R}}, \int_{0}^{L} \mathbf{r}_{x} \times \mathbf{n} d x\right\rangle
\end{aligned}
$$


Using (39) -(41), we obtain

$$
\begin{aligned}
\frac{d E}{d t}= & \left\langle\boldsymbol{\omega}_{\mathrm{R}}, I_{\mathrm{R}} \dot{\omega}_{\mathrm{R}} \times I_{\mathrm{R}} \omega_{\mathrm{R}}+\mathbf{r}(L, t) \times \mathbf{n}(L, t)\right. \\
& -\mathbf{r}(0, t) \times \mathbf{n}(0, t)+\mathbf{m}(L, t)-\mathbf{m}(0, t)\rangle \\
& +\left\langle\left(\mathbf{r}_{t}\right)_{\mathbf{B}}(L, t), \mathbf{n}(L, t)\right\rangle+\langle\mathbf{w}(L, t), \mathbf{m}(L, t)\rangle-\langle\mathbf{w}(0, t), \mathbf{m}(0, t)\rangle \\
& -\int_{0}^{L}\left\langle\left(\mathbf{r}_{x t}\right)_{\mathbf{B}}-\mathbf{w} \times \mathbf{r}_{x}, \mathbf{n}\right\rangle d x-\int_{0}^{L}\left\langle\mathbf{w}_{x}, \mathbf{m}\right\rangle d x \\
& +\int_{0}^{L}\left\langle\frac{\partial \Psi}{\partial \Gamma}, \frac{\partial \Gamma}{\partial t}\right\rangle d x+\int_{0}^{L}\left\langle\frac{\partial \Psi}{\partial \mathbf{K}}, \frac{\partial \mathbf{k}}{\partial t}\right\rangle d x
\end{aligned}
$$

Differentiating (7) with respect to time $t$ and noting that the internal energy $\Psi$ of the beam is invariant under rigid body motions (i.e., $\Psi$ measured in the body frame $\mathbf{B}$ is equal to $\Psi$ measured in the inertial frame $\mathbf{N}$-for details see, for example, Marsden 1983), we obtain

$$
\begin{aligned}
\left(\frac{\partial \mathbf{\Gamma}}{\partial t}\right)_{\mathbf{B}} & =\left(\frac{\partial}{\partial t} \Lambda^{\mathbf{T}}\right)^{\frac{\partial \mathbf{r}}{\partial x}}+\Lambda^{\mathrm{T}}\left(\frac{\partial^{2} \mathbf{r}}{\partial t \partial x}\right)_{\mathbf{B}}=-\Lambda^{\mathrm{T}} W \frac{\partial \mathbf{r}}{\partial x}+\Lambda^{\mathbf{T}}\left(\frac{\partial^{2} \mathbf{r}}{\partial t \partial x}\right)_{\mathbf{B}} \\
& =\Lambda^{\mathrm{T}}\left[\left(\mathbf{r}_{x t}\right)_{\mathbf{B}}-\mathbf{W} \times \mathbf{r}_{x}\right]
\end{aligned}
$$

where, in the second equation, we have used (3) and the skew-symmetry of $W$. Then, using the definition of the axial vector $w$ associated with $W$, we obtain (43).

Using (8), (43), (25) and (26) in (42), we obtain

$$
\begin{aligned}
\frac{d E}{d t}= & \left\langle\boldsymbol{\omega}_{\mathrm{R}}, \mathbf{r}(L, t) \times \mathbf{n}(L, t)+\mathbf{m}(L, t)+\mathbf{N}_{\mathbf{c}}(t)\right\rangle-\int_{0}^{L}\left\langle\left(\mathbf{r}_{x t}\right)_{\mathbf{B}}-\mathbf{w} \times \mathbf{r}_{x}, \mathbf{n}\right\rangle d x \\
& -\int_{0}^{L}\left\langle\mathbf{w}_{x}, \mathbf{m}\right\rangle d x+\int_{0}^{L}\left\langle\Lambda^{\mathrm{T}} \mathbf{n}, \Lambda^{\mathrm{T}}\left[\left(\mathbf{r}_{x t}\right)_{\mathbf{B}}-\mathbf{w} \times \mathbf{r}_{x}\right]\right\rangle d x+\int_{0}^{L}\left\langle\Lambda^{\mathrm{T}} \mathbf{m}, \Lambda^{\mathrm{T}} \mathbf{w}_{x}\right\rangle d x \\
& +\left\langle\left(\mathbf{r}_{t}\right)_{\mathbf{B}}(L, t), \mathbf{n}(L, t)\right\rangle+\langle\mathbf{w}(L, t), \mathbf{m}(L, t)\rangle-\langle\mathbf{w}(0, t), \mathbf{m}(0, t)\rangle
\end{aligned}
$$

Since $\Lambda$ is an orthogonal matrix, integral terms cancel each other. Also, by the boundary conditions (27), upon differentiating with respect to time $t$, at the clamped end we have:

$$
\left(\mathbf{r}_{,}(0, t)\right)_{\mathrm{B}}=0, \quad \mathbf{w}(0, t)=0, \quad t \geqslant 0
$$

Using (44) and the control law (34)-(36) in (44), we obtain

$$
\frac{d E}{d t}=-\left\langle\omega_{\mathrm{R}}, \mathbf{K} \omega_{\mathrm{R}}\right\rangle-\left\langle\left(\mathbf{r}_{t}(L, t)\right)_{\mathbf{B}}, L\left(\mathbf{r}_{t}(L, t)\right)_{\mathbf{B}}\right\rangle-\langle\mathbf{w}(L, t), \operatorname{Mw}(L, t)\rangle
$$

Since, by choice the matrices $K, \mathbf{L}, \mathbf{M}$ are positive definite, it follows from (46) that the energy $E(t)$ defined in (37) is a non-increasing function of time.

\section{Remark 1}

In the derivation of (46) we have used the non-linear equations (23)-(28) without any linearization. Furthermore, we have imposed no restrictions on the internal energy $\Psi$ of the beam, other than the assumptions that it depends on the strain measures $\boldsymbol{\Gamma}$ and $\boldsymbol{\kappa}$, and that it is invariant under rigid body motions, which is a standard assumption of the theory of elasticity (see for example Marsden 1983, 
p. 194). From this assumption it follows that the rate of change of the internal energy $\Psi$ as observed in the inertial frame $\mathbf{N}$ and as observed in the body frame $\mathbf{B}$ must be the same, since these two frames differ only by a rotation which does not depend on the spatial coordinate $x$.

To prove that the solutions of $(23)-(28)$ with the control law (34) - (36) decay to the rest state defined by $(30)-(32)$, we need to parametrize the orthogonal transformation matrix $\Lambda$ and specify the form of the internal energy function $\Psi$.

In what follows we assume that the whole motion takes place in a plane whose unit normal is the inertial axis $\mathbf{e}_{1}$. More precisely, we consider the configuration given in Fig. 1 with the following assumptions.

\section{Assumptions}

(a) The axes $\mathbf{e}_{1}, \mathbf{D}_{1}, \mathbf{d}_{1}$ coincide at all times and the rigid body may rotate only about the axis $\boldsymbol{e}_{1}$.

(b) The whole motion of the beam takes place in the plane spanned by the axes $\boldsymbol{\theta}_{2}, \mathbf{e}_{3}$.

(c) The axial deflection (i.e. along the $D_{2}$ axis) and the torsion (i.e. the rotation of the beam cross-sections about $D_{2}$ axis) are negligible.

(d) The internal energy $\Psi$ is a quadratic function of its variables, see (18).

The orthogonal transformation matrix $\Lambda$ between the body frame $\mathbf{N}$ and the frame of directors now admits representation given by (13), therefore the axial vectors $\boldsymbol{w}, \boldsymbol{\omega}, \boldsymbol{\kappa}$ can be given by (14).

Let the position vector $r=O P$ be parametrized as follows:

$$
\mathbf{r}(x, t)=(b+x) \mathbf{D}_{2}+u \mathbf{D}_{3}
$$

where $b:=|O Q|$ and $u$ is the beam displacement along $\mathbf{D}_{3}$.

With these assumptions and neglecting the higher order terms, the relevant component form of equations (23) -(28) now reduce to (cf. (19)-(22))

$$
\begin{gathered}
\mathrm{GA}\left(u_{x x}-\phi_{x}\right)=\rho u_{t t}+\rho \dot{\theta}(b+x)-\rho \dot{\theta}^{2} u \\
\mathrm{EI} \phi_{x x}+\mathrm{GA}\left(u_{x}-\phi\right)=I_{\mathbf{B}}\left(\phi_{t}+\theta\right) \\
I_{\mathrm{R}} \ddot{\theta}=b \mathrm{GA}\left[u_{x}(0, t)-\phi(0, t)\right]+\mathrm{EI} \phi_{x}(0, t)+N_{c}(t) \\
u(0, t)=0, \quad \phi(0, t)=0, \quad t \geqslant 0
\end{gathered}
$$

where $b=|\mathbf{O Q}|, \phi$ is the angle between the axes $\mathbf{d}_{2}$ and $\mathbf{D}_{2}$, and $\theta$ is the angle of rotation of the rigid body about the axis $e_{1}$; hence we have $\omega_{R}=\theta \mathbf{D}_{1}, I_{B}$ is the principal moment of inertia of the beam cross-sections about the axis $d_{1}$ and $I_{R}$ is the principal moment of inertia of the rigid body about the axis $D_{1}$.

The component form of the natural control law (34)-(36) now becomes

$$
\begin{gathered}
\mathrm{GA}\left[u_{x}(L, t)-\phi((L, t)]+\alpha u_{t}(L, t)=0\right. \\
\mathrm{EI} \phi_{x}(L, t)+\beta \phi_{t}(L, t)=0 \\
N_{\mathrm{c}}(t)=-(b+L) \mathrm{GA}\left[u_{x}(L, t)-\phi(L, t)\right]-\mathrm{EI} \phi_{x}(L, t)-k \theta
\end{gathered}
$$

where $k>0, \alpha>0$, and $\beta>0$ are arbitrary positive numbers. 
The total energy $E(t)$ given by (37), now becomes

$$
\begin{aligned}
E(t)= & \frac{1}{2} I_{\mathrm{R}} \theta^{2}+\frac{1}{2} \int_{0}^{L} \rho\left\langle\mathbf{r}_{t}, \mathbf{r}_{t}\right\rangle d x+\frac{1}{2} \int_{0}^{L} I_{\mathrm{B}}\left(\phi_{t}+\theta\right)^{2} d x \\
& +\frac{1}{2} \int_{0}^{L} \mathrm{GA}\left(u_{x}-\phi\right)^{2} d x+\frac{1}{2} \int_{0}^{L} \mathrm{El} \phi_{x}^{2} d x
\end{aligned}
$$

and the rate of change of $E(t)$ given by (46) now reduces to

$$
\frac{d E}{d t}=-k \theta^{2}-\alpha u_{t}^{2}(L, t)-\beta \phi_{t}^{2}(L, t)
$$

\section{Theorem 1}

Consider the system given by (48) - (51) together with the control law (52)(54). Then there exists a $T \geqslant 0$ such that for $t \geqslant T$, the energy $E(t)$ given by (37) decays as $O(1 / t)$.

\section{Remark 2}

Equations (48) $-(51)$ are the component forms of equations (23) - (28) under the assumptions $(a)-(d)$ stated after (46). As a result of these assumptions, (48) $-(51)$ represent the equations of motion for the planar motion of a rigid body whose centre of mass is fixed in an inertial frame, with a beam modelled as a Timoshenko beam clamped to it.

\section{Remark 3}

If we use the conclusion of Theorem $1,(55)$ and (56), then we see that the solutions of $(48)-(51)$ tend to the rest state defined by $(30)-(32)$ as $t \rightarrow \infty$.

\section{Proof of Theorem 1}

We define the following function $V(t)$ :

$$
\begin{aligned}
V(t)= & 2(1-\varepsilon) t E(t)+2 \int_{0}^{L} \rho x\left(u_{t}+\theta(b+x)\right) u_{x} d x+2 \int_{0}^{L} I_{\mathbf{B}} x \phi_{x}\left(\phi_{t}+\theta\right) d x \\
& +\delta \int_{0}^{L} I_{\mathbf{B}} \phi\left(\phi_{t}+\theta\right) d x-\delta \int_{0}^{L} \rho u\left[u_{t}+\theta(b+x)\right] d x
\end{aligned}
$$

where $\varepsilon \in(0,1)$ and $\delta>0$ are constants yet to be determined.

To prove the theorem, first show that there exists a constant $C>0$ such that the following estimate holds for all $t \geqslant 0$ :

$$
[2(1-\varepsilon) t-C] E(t) \leqslant V(t) \leqslant[2(1-\varepsilon) t+C] E(t)
$$

then we prove that there exists a $T_{1} \geqslant 0$ such that

$$
\frac{d V}{d t} \leqslant 0, \quad t \geqslant T_{1}
$$


Combining (58) and (59) we obtain

$$
E(t) \leqslant \frac{V\left(T_{1}\right)}{2(1-\varepsilon) t-C}, \quad t>T
$$

where $T=\max \left\{T_{1}, \frac{C}{2(1-\varepsilon)}\right\}$.

Since $E(t)$ is a non-increasing function of time by (56), from (58) it follows that $V\left(T_{1}\right)<\infty$, and (60) proves that for sufficiently large $t, E(t)$ decays as $O(1 / t)$.

Owing to the boundary conditions $u(0, t)=0, \phi(0, t)=0$ for all $t \geqslant 0$, we obtain the following estimates which follow from the Jensen inequality (see for example Royden 1968).

$$
\phi^{2}(x, t) \leqslant L \int_{0}^{L} \phi_{s}^{2} d x, \quad u^{2}(x, t) \leqslant L \int_{0}^{L} u_{s}^{2} d s \quad x \in[0, L]
$$

Using (61) we obtain the following estimate:

$$
\int_{0}^{L} u_{x}^{2} d x=\int_{0}^{L}\left(u_{x}-\phi+\phi\right)^{2} d x \leqslant 2 \int_{0}^{L}\left(u_{x}-\phi\right)^{2} d x+2 L^{2} \int_{0}^{L} \phi_{x}^{2} d x
$$

For simplicity, we define the quantities $A_{1}, A_{2}, A_{3}$ and $A_{4}$, which appear in (57), as follows:

$$
\begin{aligned}
& A_{1}:=2 \int_{0}^{L} \rho x\left[u_{t}+\theta(b+x)\right] u_{x} d x, \quad A_{2}:=2 \int_{0}^{L} I_{\mathbf{B}} x \phi_{x}\left(\phi_{t}+\theta\right) d x \\
& A_{3}:=\delta \int_{0}^{L} I_{\mathbf{B}} \phi\left(\phi_{t}+\dot{\theta}\right) d x, \quad A_{4}:=-\delta \int_{0}^{L} \rho u\left[u_{t}+\theta(b+x)\right] d x
\end{aligned}
$$

Using (47) in (29), we obtain

$$
\left(\frac{\partial r}{\partial t}\right)_{\mathbf{N}}=-\theta u \mathbf{D}_{2}+\left[u_{t}+\theta(b+x)\right] \mathbf{D}_{3}
$$

Using (61), (62) and (65), we obtain the following estimates:

$$
\begin{aligned}
\left|A_{1}\right| & \leqslant \rho L \int_{0}^{L} u_{x}^{2} d x+\rho L \int_{0}^{L}\left[u_{t}+\theta(b+x)\right]^{2} d x \\
& \leqslant 2 \rho L \int_{0}^{L}\left(u_{x}-\phi\right)^{2} d x+2 \rho L^{3} \int_{0}^{L} \phi_{x}^{2} d x+L \int_{0}^{L} \rho<\mathbf{r}_{t}, \mathbf{r}_{1}>d x \leqslant K_{1} E(t)
\end{aligned}
$$

where

$$
\begin{gathered}
K_{1}=2 \frac{\max \left\{2 \rho L, 2 \rho L^{3}, L\right\}}{\min \left\{I_{\mathrm{R}}, 1, \mathrm{GA}, \mathrm{EI}\right\}} \\
\left|A_{2}\right| \leqslant I_{\mathrm{B}} L \int_{0}^{L} \phi_{x}^{2} d x+I_{\mathbf{B}} \mathrm{L} \int_{0}^{L}\left(\phi_{t}+\theta\right)^{2} d x \leqslant K_{2} E(t)
\end{gathered}
$$

where

$$
\begin{gathered}
K_{2}=2 \frac{\max \left\{I_{\mathrm{B}} L, L\right\}}{\min \left\{I_{\mathrm{R}}, 1, \mathrm{GA}, \mathrm{EI}\right\}} \\
\left|A_{3}\right| \leqslant \delta I_{\mathrm{B}} \int_{0}^{L} \phi^{2} d x+\delta I_{\mathrm{B}} \int_{0}^{L}\left(\phi_{t}+\theta\right)^{2} d x \\
\leqslant \delta I_{\mathrm{B}} L^{2} \int_{0}^{L} \phi_{x}^{2} d x+\delta I_{\mathrm{B}} \int_{0}^{L}\left(\phi_{t}+\theta\right)^{2} d x \leqslant K_{3} E(t)
\end{gathered}
$$


where

$$
\begin{gathered}
K_{3}=2 \frac{\max \left\{\delta I_{\mathrm{B}} L^{2}, \delta\right\}}{\min \left\{I_{\mathrm{R}}, 1, \mathrm{GA}, \mathrm{EI}\right\}} \\
\left|A_{4}\right| \leqslant \delta \rho \int_{0}^{L} u^{2} d x+\delta \rho L^{2} \int_{0}^{L}\left[u_{t}+\theta(b+x)\right]^{2} d x \\
\leqslant 2 \delta \rho L^{2} \int_{0}^{L}\left(u_{x}-\phi\right)^{2} d x+2 \delta \rho L^{4} \int_{0}^{L} \phi_{x}^{2} d x+\delta \int_{0}^{L} \rho\left\langle\mathrm{r}_{t}, \mathbf{r}_{t}\right\rangle d x \\
\leq K_{4} E(t)
\end{gathered}
$$

where

$$
K_{4}=2 \frac{\max \left\{2 \delta \rho L_{2}, 2 \delta \rho L^{4}, \delta\right\}}{\min \left\{I_{\mathrm{R}}, 1, \mathrm{GA}, \mathrm{EI}\right\}}
$$

Using (66) -(69) in (57), we obtain (58) with $C=K_{1}+K_{2}+K_{3}+K_{4}$.

To prove (59), we first differentiate $A_{1}$ with respect to time

$$
\begin{aligned}
\frac{d A_{1}}{d t}= & 2 \int_{0}^{L} \rho x\left[u_{t}+\theta(b+x)\right] u_{x t} d x+2 \int_{0}^{L} \rho x\left[u_{t t}+\dot{\theta}(b+x)\right] u_{x} d x \\
= & 2 \int_{0}^{L} \rho x u_{t} u_{x t} d x+2 \int_{0}^{L} \rho x u_{x t} \theta(b+x) d x \\
& +2 \int_{0}^{L} \mathrm{GA} x u_{x}\left(u_{x x}-\phi_{x}\right) d x+2 \theta^{2} \int_{0}^{L} \rho x u u_{x} d x \\
= & \rho L u_{t}^{2}(L, t)-\int_{0}^{L} \rho u_{t}^{2} d x+2\left[\rho L(b+L) u_{t}(L, t)-\int_{0}^{L} \rho(b+2 x) u_{t} d x\right] \theta \\
& +\mathrm{GA}\left[L u_{x}^{2}(L, t)-\int_{0}^{L} u_{x}^{2} d x-2 \int_{0}^{L} x u_{x} \phi_{x} d x\right] \\
& +\left[\rho L u^{2}(L, t)-\rho \int_{0}^{L} u^{2} d x\right] \theta^{2}
\end{aligned}
$$

where, in the second equation, we used (48). Then, using integration by parts and the fact that $\theta$ does not depend on $x$, we obtain (70).

Since $\theta(\cdot)$ does not depend on $x, A_{2}$ is equivalent to the following:

$$
A_{2}=2 \int_{0}^{L} I_{\mathrm{B}} x(\phi+\theta)_{x}(\phi+\theta)_{t} d t
$$

Upon differentiating (71) with respect to time, we obtain

$$
\begin{aligned}
\frac{d A_{2}}{d t}= & 2 \int_{0}^{L} I_{\mathbf{B}} x(\phi+\theta)_{x t} d x+2 \int_{0}^{L} x I_{\mathbf{B}}\left(\phi_{t t}+\theta\right)(\phi+\theta)_{x} d x \\
= & I_{\mathrm{B}} L\left(\phi_{t}(L, t)+\theta\right)^{2}-\int_{0}^{L} I_{\mathbf{B}}\left(\phi_{t}+\theta\right)^{2} d x \\
& +2 \int_{0}^{L} \mathrm{EI} x \phi_{x} \phi_{x x} d x+2 \int_{0}^{L} \mathrm{GA} x \phi_{x}\left(u_{x}-\phi\right) d x \\
= & I_{\mathbf{B}} L\left(\phi_{t}(L, t)+\theta\right)^{2}-\int_{0}^{L} I_{\mathbf{B}}\left(\phi_{t}+\theta\right)^{2} d x+\mathrm{EI} L \phi_{x}^{2}(L, t) \\
& -\int_{0}^{L} \mathrm{EI} \phi_{x}^{2} d x+2 \mathrm{GA} \int_{0}^{L} x \phi_{x} u_{x} d x-\mathrm{GA} L \phi^{2}(L, t)+\mathrm{GA} \int_{0}^{\mathrm{L}} \phi^{2} d x
\end{aligned}
$$


where, in the second equation, we have used integration by parts and (49). Then, again using integration by parts, we obtain (72).

Upon differentiating $A_{3}$, we obtain

$$
\begin{aligned}
\frac{d A_{3}}{d t}= & \delta \int_{0}^{L} I_{\mathbf{B}}\left(\phi_{t}+\theta\right)^{2} d x-\delta \int_{0}^{L} I_{\mathbf{B}} \theta\left(\phi_{t}+\theta\right) d x+\delta \int_{0}^{L} I_{\mathrm{B}} \phi\left(\phi_{t t}+\theta\right) d x \\
= & \delta \int_{0}^{L} I_{\mathbf{B}}\left(\phi_{t}+\theta\right)^{2} d x-\delta \int_{0}^{L} I_{\mathrm{B}} \theta\left(\phi_{t}+\theta\right) d x \\
& +\delta \int_{0}^{L} \phi\left[\mathrm{EI} \phi_{x x}+\mathrm{GA}\left(u_{x}-\phi\right)\right] d x \\
= & \delta \int_{0}^{L} I_{\mathrm{B}}\left(\phi_{t}+\theta\right)^{2} d x-\delta \int_{0}^{L} I_{\mathrm{B}} \theta\left(\phi_{t}+\theta\right) d x \\
& +\delta \mathrm{EI} \phi(L, t) \phi_{x}(L, t)-\delta \mathrm{EI} \int_{0}^{L} \phi_{x}^{2} d x \\
& +\delta \mathrm{GA} \phi(L, t) u(L, t)-\delta \mathrm{GA} \int_{0}^{L} \phi_{x} u d x-\delta \mathrm{GA} \int_{0}^{L} \phi^{2} d x
\end{aligned}
$$

where in the first equation we have added and subtracted $\theta$; in the second equation we have used (49). Then, using integration by parts and the boundary conditions (51), we obtain (73).

Similarly, upon differentiating $A_{4}$, we obtain

$$
\begin{aligned}
\frac{d A_{4}}{d t}= & -\delta \int_{0}^{L} \rho u_{t}\left[u_{t}+\theta(b+x)\right] d x-\delta \int_{0}^{L} \rho u\left[u_{t t}+\ddot{\theta}(b+x)\right] d x \\
= & -\delta \int_{0}^{L} \rho u_{t}^{2} d x-\delta \theta \int_{0}^{L} \rho(b+x) u_{t} d x \\
& -\delta \int_{0}^{L} u\left[\mathrm{GA}\left(u_{x x}-\phi_{x}\right)\right] d x-\delta \theta^{2} \int_{0}^{L} \rho u^{2} d x \\
= & -\delta \int_{0}^{L} \rho u_{t}^{2} d x-\delta \theta \int_{0}^{L} \rho(b+x) u_{t} d x-\delta \mathrm{GA} u(L, t) u_{x}(L, t) \\
& +\delta \mathrm{GA} \int_{0}^{L} u_{x}^{2} d x+\delta \mathrm{GA} \int_{0}^{L} u \phi_{x} d x-\delta \theta^{2} \int_{0}^{L} \rho u^{2} d x
\end{aligned}
$$

where, in the second equation (48) was used. Then, integrating by parts and using the boundary conditions (51), we obtain (74). 
Differentiating $V(t)$ with respect to time and using $(70)-(74)$, we obtain the following:

$$
\begin{aligned}
& \frac{d V}{d t}=2(1-\varepsilon) t \frac{d E}{d t}+2(1-\varepsilon) E(t)+\sum_{i=1}^{4} \frac{d A_{i}}{d t} \\
& =-2(1-\varepsilon) k t \theta^{2}-2(1-\varepsilon) \alpha t u_{t}^{2}(L, t)-2(1-\varepsilon) \beta t \phi_{t}^{2}(L, t)+(1-\varepsilon) I_{\mathrm{R}} \theta^{2} \\
& +(1-\varepsilon) \int_{0}^{L} \rho\left\langle\mathbf{r}_{t}, \mathbf{r}_{t}\right\rangle d x+(1-\varepsilon) \int_{0}^{L} I_{\mathbf{B}}\left(\phi_{t}+\theta\right)^{2} d x \\
& +(1-\varepsilon) \mathrm{GA} \int_{0}^{L}\left(u_{x}-\phi\right)^{2} d x \\
& +(1-\varepsilon) \mathrm{EI} \int_{0}^{L} \phi_{x}^{2} d x+\rho L u_{f}^{2}(L, t)-\int_{0}^{L} \rho u_{t}^{2} d x+\mathrm{GA} L u_{x}^{2}(L, t) \\
& +2\left[\rho L(b+L) u_{t}(L, t)-\int_{0}^{L} \rho(b+2 x) u_{t} d x\right] \dot{\theta} \\
& -\mathrm{GA} \int_{0}^{L} u_{x}^{2} d x-2 \mathrm{GA} \int_{0}^{L} x u_{x} \phi_{x} d x \\
& +\rho\left[L u^{2}(L, t)-\int_{0}^{L} u^{2} d x\right] \theta^{2}+I_{8} L\left(\phi_{l}(L, t)+\theta\right)^{2} \\
& -\int_{0}^{L} I_{\mathbf{B}}\left(\phi_{t}+\theta\right)^{2} d x+\operatorname{EI} L \phi_{x}^{2}(L, t) \\
& -\int_{0}^{L} \mathrm{EI} \phi_{x}^{2} d x+2 \mathrm{GA} \int_{0}^{L} x \phi_{x} u_{x} d x-\mathrm{GA} L \phi^{2}(L, t) \\
& +\mathrm{GA} \int_{0}^{L} \phi^{2} d x+\delta \int_{0}^{L} I_{\mathbf{B}}\left(\phi_{t}+\theta\right)^{2} d x \\
& -\delta \int_{0}^{L} I_{\mathrm{B}} \theta(\phi,+\theta) d x+\delta \operatorname{EI} \phi(L, t) \phi_{x}(L, t) \\
& -\delta \mathrm{EI} \int_{0}^{L} \phi_{x}^{2} d x-\delta \mathrm{GA} \int_{0}^{L} \phi^{2} d x \\
& +\delta \mathrm{GA} \phi(L, t) u(L, t)-\delta \mathrm{GA} \int_{0}^{L} \phi_{x} u d x-\delta \int_{0}^{L} \rho u_{t}^{2} d x \\
& -\delta \theta \int_{0}^{L} \rho(b+x) u_{t} d x-\delta \mathrm{GA} u(L, t) u_{x}(L, t) \\
& +\delta \mathrm{GA} \int_{0}^{L} u_{x}^{2} d x+\delta \mathrm{GA} \int_{0}^{L} u \phi_{x} d x-\delta \theta^{2} \int_{0}^{L} \rho u^{2} d x
\end{aligned}
$$

where in the first equation we have used (57) and (63), (64). Then, using (56), (55) and $(70)-(74)$, we obtain (75).

Using (65), the integral associated with the inner product $\left\langle\mathbf{r}_{t}, \boldsymbol{r}_{t}\right\rangle$ can be written as

$$
\begin{array}{r}
\int_{0}^{L}\left\langle\rho \mathbf{r}_{t}, \mathbf{r}_{t}\right\rangle d x=\theta^{2} \int_{0}^{L} \rho u^{2} d x+\int_{0}^{L} \rho u_{t}^{2} d x+2 \theta \int_{0}^{L} \rho(b+x) u_{t} d x \\
+\theta^{2} \int_{0}^{L}(b+x)^{2} d x
\end{array}
$$


After cancellations, using (76) and collecting likewise terms, (75) becomes

$$
\begin{aligned}
\frac{d V}{d t}= & -\left[2(1-\varepsilon) k t-(1-\varepsilon) I_{\mathrm{R}}-\int_{0}^{L} \rho\left(u^{2}+(b+x)^{2}\right) d x-\rho L u^{2}(L, t)-\int_{0}^{L} \rho u^{2} d x\right. \\
& \left.+\delta \int_{0}^{L} \rho u^{2} d x\right] \theta^{2}-[\varepsilon+\delta] \int_{0}^{L} \rho u_{t}^{2} d x-[\varepsilon+\delta] \\
& \times \int_{0}^{L} \mathrm{EI} \phi_{x}^{2} d x-[\varepsilon-\delta] \int_{0}^{L} I_{\mathrm{B}}\left(\phi_{t}+\theta\right)^{2} d x \\
& +(\delta-1) \mathrm{GA} \int_{0}^{L} u_{x}^{2} d x+(1-\delta) \mathrm{GA} \int_{0}^{L} \phi^{2} d x+(1-\varepsilon) \mathrm{GA} \int_{0}^{L}\left(u_{x}-\phi\right)^{2} d x \\
& -[2(1-\varepsilon)-\delta] \theta \int_{0}^{L} \rho(b+x) u_{t} d x-\delta \int_{0}^{L} I_{\mathbf{B}} \theta\left(\phi_{t}+\theta\right) d x \\
& -[2(1-\varepsilon) \alpha t-\rho L] u_{t}^{2}(L, t)-2(1-\varepsilon) \beta t \phi_{t}^{2}(L, t) \\
& +2\left[\rho L(b+L) u_{t}(L, t)-\rho \int_{0}^{L}(b+2 x) u_{t} d x\right] \theta+\mathrm{GA} L u_{x}^{2}(L, t) \\
& +I_{\mathrm{B}} L(\phi,(L, t)+\theta)^{2}+\mathrm{EI} L \phi_{x}^{2}(L, t)-\mathrm{GA} L \phi^{2}(L, t) \\
& +\delta \mathrm{EI} \phi(L, t) \phi_{x}(L, t)-\delta \mathrm{GA}\left[\left(u_{x}(L, t)-\phi(L, t)\right] u(L, t)\right.
\end{aligned}
$$

Using the following simple inequalities:

$$
\begin{gathered}
a b \leqslant \delta^{2} a^{2}+\frac{b^{2}}{\delta^{2}} \quad a, b, \delta \in \mathbb{R} \delta \neq 0 \\
(a+b)^{2} \leqslant 2\left(a^{2}+b^{2}\right) \quad a, b \in \mathbb{R}
\end{gathered}
$$

the boundary controls (52), (53) and the fact that $E(t) \leqslant E(0)$ (see (56)), we obtain the following estimates for some terms appearing in (77):

$$
\int_{0}^{L} u^{2} d x \leqslant L^{2} \int_{0}^{L} u_{x}^{2} d x \leqslant 2 L^{2} \int_{0}^{L}\left(u_{x}-\phi\right)^{2} d x+2 L^{4} \int_{0}^{L} \phi_{x}^{2} d x \leqslant M_{1} E(0)
$$

where

$$
\begin{gathered}
M_{1}=2 \frac{\max \left\{2 L^{2}, 2 L^{4}\right\}}{\min \left\{I_{\mathrm{R}}, 1, \mathrm{GA}, \mathrm{EI}\right\}} \\
u^{2}(L, t) \leqslant M_{2} E(0)
\end{gathered}
$$

where $M_{2}=M_{1} / L($ see $(61),(62))$

$$
\begin{gathered}
\int_{0}^{L} \phi^{2} d x \leqslant L^{2} \int_{0}^{L} \phi_{x}^{2} d x \\
\int_{0}^{L}\left(u_{x}-\phi\right)^{2} d x \leqslant 2 \int_{0}^{L} u_{x}^{2} d x+2 \int_{0}^{L} \phi^{2} d x \leqslant 2 \int_{0}^{L} u_{x}^{2} d x \\
+2 L^{2} \int_{0}^{L} \phi_{x}^{2} d x \\
\int_{0}^{L} \rho(b+x) u_{t} \theta d x \leqslant \delta_{1}^{2} \int_{0}^{L} \rho u_{t}^{2} d x+\frac{\rho \int_{0}^{L}(b+x)^{2} d x}{\delta_{1}^{2}} \theta^{2}
\end{gathered}
$$




$$
\begin{array}{cc}
\int_{0}^{L} I_{\mathrm{B}} \theta\left(\phi_{t}+\theta\right) d x \leqslant \delta_{2}^{2} \int_{0}^{L} I_{\mathrm{B}}\left(\phi_{t}+\theta\right)^{2} d x+\frac{I_{\mathrm{B}} L}{\delta_{2}^{2}} \theta^{2} & \text { (by (78)) } \\
u_{t}(L, t) \theta \leqslant \delta_{3}^{2} u_{t}^{2}(L, t)+\frac{1}{\delta_{3}^{2}} \theta^{2} & \text { (by (78)) } \\
\int_{0}^{L} \rho(b+2 x) u_{t} d x \leqslant \delta_{4}^{2} \int_{0}^{L} \rho u_{t}^{2} d x+\frac{\rho \int_{0}^{L}(b+2 x)^{2} d x}{\delta_{4}^{2}} \hat{\theta}^{2} & \text { (by (78)) }
\end{array}
$$

GA $L u_{x}^{2}(L, t)=\mathrm{GA} L \phi^{2}(L, t)-2 \alpha L \phi(L, t) u_{t}(L, t)$

$$
\begin{gathered}
+\frac{\alpha^{2} L}{\mathrm{GA}} u_{t}^{2}(L, t) \leqslant \mathrm{GA} L \phi^{2}(L, t) \\
+2 \alpha L^{2} \delta_{s}^{2} \int_{0}^{L} \phi_{x}^{2} d x+\left(\frac{2 \alpha^{2} L}{\delta_{5}^{2}}+\frac{\alpha^{2} L}{\mathrm{GA}}\right) u_{t}^{2}(L, t) \quad(\text { by }(52)) \\
I_{\mathrm{B}} L\left(\phi_{t}(L, t)+\theta\right)^{2} \leqslant 2 I_{\mathrm{B}} L \phi_{t}^{2}(L, t)+2 I_{\mathrm{B}} L \theta^{2} \\
\mathrm{EI} \phi_{x}^{2}(L, t) \leqslant \frac{\beta^{2}}{\mathrm{EI}} \phi_{t}^{2}(L, t)
\end{gathered}
$$

$\delta \mathrm{EI} \phi(L, t) \phi_{x}(L, t)=-\delta \beta \phi(L, t) \phi_{t}(L, t) \leqslant \delta \beta \delta_{6}^{2} \phi^{2}(L, t)+\frac{\delta \beta}{\delta_{6}^{2}} \phi_{t}^{2}(L, t)$

$$
\leqslant \delta \beta L \delta_{6}^{2} \int_{0}^{L} \phi_{x}^{2} d x+\frac{\delta \beta}{\delta_{6}^{2}} \phi_{t}^{2}(L, t) \quad \text { (by (78), (61)) }
$$

$-\delta \mathrm{GA}\left[u_{x}(L, t)-\phi(L, t)\right] u(L, t)=\delta \alpha u_{t}(L, t) u(L, t)$

$$
\leqslant \delta \alpha L \delta_{7}^{2} \int_{0}^{L} u_{x}^{2} d x+\frac{\delta \alpha}{\delta_{7}^{2}} u_{t}^{2}(L, t) \quad(\text { by }(52))
$$

where $\delta_{i}, i=1, \ldots, 7$, are any non-zero real numbers.

Using the estimates $(80)-(92)$ in (77), the latter becomes

$$
\begin{aligned}
\frac{d V}{d t} \leqslant & -\left[2(1-\varepsilon) k t-D_{1}\right] \theta^{2}(t)-\left[\varepsilon+\delta-(2(1-\varepsilon)) \delta_{1}^{2}-2 \delta_{4}^{2}\right] \int_{0}^{L} \rho u_{t}^{2} d x \\
& -\left[2(1-\varepsilon) \alpha t-D_{2}\right] u_{t}^{2}(L, t)-\left[2(1-\varepsilon) \beta t-D_{3}\right] \phi_{t}^{2}(L, t) \\
& -\left[\varepsilon+\delta-(3-\delta-2 \varepsilon) \mathrm{GA} L^{2}-2 \alpha L^{2} \delta_{5}^{2}-\delta \beta L \delta_{6}^{2}\right] \int_{0}^{L} \phi_{x}^{2} d x \\
& -\left[\varepsilon-\delta \delta_{2}^{2}\right] \int_{0}^{L} I_{\mathrm{B}}\left(\phi_{t}+\theta\right)^{2} d x-\left[(2 \varepsilon-\delta) \mathrm{GA}-\delta \alpha \delta_{7}^{2}\right] \int_{0}^{L} u_{x}^{2} d x
\end{aligned}
$$


where

$$
\begin{aligned}
D_{1}= & (1-\varepsilon) I_{\mathrm{R}}+(2 \rho+\delta) M_{1}+\frac{I_{\mathrm{B}}}{\delta_{2}^{2}}+\rho L M_{2}+2 I_{\mathrm{B}} L+\frac{2 L(b+L)}{\delta_{3}^{2}} \\
& +\rho \int_{0}^{L}(b+x)^{2} d x+\frac{(2(1-\varepsilon)-\delta) \rho \int_{0}^{L}(b+x)^{2} d x}{\delta_{1}^{2}}+\frac{2 \rho \int_{0}^{L}(b+2 x)^{2} d x}{\delta_{4}^{2}} \\
D_{2}= & \rho L+2 \rho L(b+L) \delta_{3}^{2}+\frac{2 \alpha L}{\delta_{5}^{2}}+\frac{\delta^{2} L}{\mathrm{GA}}+\frac{\delta \alpha}{\delta_{7}^{2}} \\
D_{3}= & 2 I_{\mathrm{B}} L+\frac{\beta^{2}}{\mathrm{EI}}+\frac{\delta \beta}{\delta_{6}^{2}}
\end{aligned}
$$

By choosing $\varepsilon$ and $\delta$ sufficiently close to but smaller than 1 and by choosing $\delta_{i}$, $i=1, \ldots, 7$, small enough, each term multiplying the integral terms in (93) can be made negative. To see this, define $\hat{\varepsilon}$ and $\delta$ as follows:

$$
\hat{\varepsilon}:=1-\varepsilon, \quad \delta:=1-\delta
$$

Then sufficient conditions to make the coefficients of the integral terms in (93) negative are

$$
\left(1+\mathrm{GA} L^{2}\right) \hat{\delta}+\left(1+2 \mathrm{GA} L^{2}\right) \hat{\varepsilon}<2, \quad 2 \hat{\varepsilon}<\delta<1
$$

It is easy to see that one can find $\hat{\varepsilon}$ and $\delta$ sufficiently small to satisfy (98) (e.g. choose $\hat{\varepsilon}=1 / 8\left(1+2 \mathrm{GA} L^{2}\right)$ and $\left.\delta=1 / 2\left(1+2 \mathrm{GA} L^{2}\right)\right)$. Then, choosing $\delta_{i}$, $i=1, \ldots, 7$, small enough, the coefficients of each integral term in (93) become negative. Then, from (93) it follows that (59) holds with

$$
T_{1}=\max \left\{\frac{D_{1}}{2(1-\varepsilon) k}, \frac{D_{2}}{2(1-\varepsilon) \alpha}, \frac{D_{2}}{2(1-\varepsilon) \beta}\right.
$$

Then the argument following (59) proves Theorem 1.

\section{Existence, uniqueness and exponential decay of solutions}

In the previous section, we proved that the solutions of the equations of motion, i.e. (48)-(51), decay at least as $O(1 / t)$ for large $t$. In this section we establish an existence and uniqueness theorem for the solutions of the equations mentioned above, and then prove that solutions actually decay exponentially.

We repeat the equations of motion studied in the previous section; namely (48) $-(51)$, for all $t \geqslant 0, x \in(0, L)$ :

$$
\begin{aligned}
u_{t}= & \frac{\mathrm{GA}}{\rho}\left(u_{x x}-\phi_{x}\right)+\frac{\mathrm{GA}}{I_{\mathrm{R}}}(b+x) \int_{0}^{L}(b+x)\left(u_{x x}-\phi_{x}\right) d x \\
& -\frac{\mathrm{GA}}{I_{\mathrm{R}}}(b+x) \int_{0}^{L}\left(u_{x}-\phi\right) d x \\
& +\frac{\mathrm{EI}}{I_{\mathrm{R}}}(b+x) \int_{0}^{L} \phi_{x x} d x+k(b+x) \theta+\rho \theta^{2} u
\end{aligned}
$$




$$
\begin{gathered}
\phi_{t}=\frac{\mathrm{EI}}{I_{\mathrm{B}}} \phi_{x x}+\frac{\mathrm{GA}}{I_{\mathrm{B}}}\left(u_{x}-\phi\right)+\frac{\mathrm{GA}}{I_{\mathrm{R}}} \int_{0}^{L}(b+x)\left(u_{x x}-\phi_{x}\right) d x \\
-\frac{\mathrm{GA}}{I_{\mathrm{R}}} \int_{0}^{L}\left(u_{x}-\phi\right) d x+\frac{\mathrm{EI}}{I_{\mathrm{R}}} \int_{0}^{L} \phi_{x x} d x+k \dot{\theta} \\
\dot{\theta}=-\frac{\mathrm{GA}}{I_{\mathrm{R}}} \int_{0}^{L}(b+x)\left(u_{x x}-\phi_{x}\right) d x \\
+\frac{\mathrm{GA}}{I_{\mathrm{R}}} \int_{0}^{L}\left(u_{x}-\phi\right) d x-\frac{\mathrm{EI}}{I_{\mathrm{R}}} \int_{0}^{L} \phi_{x x} d x-k \theta \\
u(0, t)=0, \quad \phi(0, t)=0 \\
\mathrm{GA}\left[u_{x}(L, t)-\phi(L, t)\right]+\alpha u_{t}(L, t)=0 \\
\mathrm{EI} \phi_{x}(L, t)+\beta \phi_{t}(L, t)=0
\end{gathered}
$$

We define the function space $\mathscr{H}$ in which the solutions of (99)-(104) evolve, as follows:

$$
\mathscr{H}:=\left\{(u, u, \phi \phi, \theta)^{\mathrm{T}} \mid u \in \mathbf{H}_{0}^{1}, \phi \in \mathbf{H}_{0}^{1}, u_{t} \in \mathbf{L}^{2}, \phi_{t} \in \mathbf{L}^{2}, \theta \in \mathbb{R}\right\}
$$

where the spaces $\mathbf{L}^{2}$ and $\mathbf{H}_{0}^{k}$ are defined as follows:

$$
\begin{gathered}
\mathbf{L}^{2}=\left\{f:[0, L] \rightarrow \mathbf{R} \mid \int_{0}^{L} f^{2} d x<\infty\right\} \\
\mathbf{H}_{0}^{k}=\left\{f \in \mathbf{L}^{2} \mid f, f^{\prime}, f^{\prime \prime}, \ldots, f^{(k)} \in \mathbf{L}^{2}, f(0)=0\right\}
\end{gathered}
$$

Equations (99)-(104) can be written in the following abstract form:

$$
\dot{z}=A z+g(z), \quad z(0) \in \mathscr{H}
$$

where $z=(u u, \phi \phi, \theta)^{\mathbf{T}} \in \mathscr{H}$ and the operator $A: \mathscr{H} \rightarrow \mathscr{H}$ is a linear unbounded operator whose matrix form is specified as follows:

$$
A=\left\{m_{i j}: i, j=1, \ldots, 6\right\}
$$

where all $m_{i j}$ are zero except

$$
\begin{aligned}
m_{12}= & m_{34}=1 \quad m_{25}=k(b+x) \quad m_{45}=k \quad m_{55}=-k \\
m_{21}= & \frac{\mathrm{GA}}{I_{\mathrm{R}}} \frac{\partial^{2}}{\partial x^{2}}+\frac{\mathrm{GA}}{I_{\mathrm{R}}}(b+x) \int_{0}^{L}(b+x) \frac{\partial^{2}}{\partial x^{2}} d x-\frac{\mathrm{GA}}{I_{\mathrm{R}}}(b+x) \int_{0}^{L} \frac{\partial}{\partial x} d x \\
m_{23}= & -\frac{\mathrm{GA}}{\rho} \frac{\partial}{\partial x}-\frac{\mathrm{GA}}{I_{\mathrm{R}}}(b+x) \int_{0}^{L}(b+x) \frac{\partial}{\partial x} d x \\
& +\frac{\mathrm{GA}}{I_{\mathrm{R}}}(b+x) \int_{0}^{L} d x+\frac{\mathrm{EI}}{I_{\mathrm{R}}}(b+x) \int_{0}^{L} \frac{\partial^{2}}{\partial x^{2}} d x \\
m_{41}= & \frac{\mathrm{GA}}{I_{\mathrm{B}}} \frac{\partial}{\partial x}+\frac{\mathrm{GA}}{I_{\mathrm{R}}} \int_{0}^{L}(b+x) \frac{\partial^{2}}{\partial x^{2}} d x-\frac{\mathrm{GA}}{I_{\mathrm{R}}} \int_{0}^{L} \frac{\partial}{\partial x} d x \\
m_{43}= & \frac{\mathrm{EI}}{I_{\mathbf{B}}} \frac{\partial^{2}}{\partial x^{2}}-\frac{\mathrm{GA}}{I_{\mathbf{B}}}-\frac{\mathrm{GA}}{I_{\mathrm{R}}} \int_{0}^{L}(b+x) \frac{\partial}{\partial x} d x+\frac{\mathrm{GA}}{I_{\mathrm{R}}} \int_{0}^{L} d x+\frac{\mathrm{EI}}{I_{\mathrm{R}}} \int_{0}^{L} \frac{\partial^{2}}{\partial x^{2}} d x
\end{aligned}
$$




$$
\begin{aligned}
& m_{51}=-\frac{\mathrm{GA}}{I_{\mathrm{R}}} \int_{0}^{L}(b+x) \frac{\partial^{2}}{\partial x^{2}} d x+\frac{\mathrm{GA}}{I_{\mathrm{R}}} \int_{0}^{L} \frac{\partial}{\partial x} d x \\
& m_{53}=\frac{\mathrm{GA}}{I_{\mathrm{R}}} \int_{0}^{L}(b+x) \frac{\partial}{\partial x} d x-\frac{\mathrm{GA}}{I_{\mathrm{R}}} \int_{0}^{L} d x-\frac{\mathrm{EI}}{I_{\mathrm{R}}} \int_{0}^{L} \frac{\partial^{2}}{\partial x^{2}} d x
\end{aligned}
$$

the operator $g: \mathscr{H} \rightarrow \mathscr{H}$ is a non-linear operator defined as

$$
g(z)=\left(g_{1}, \ldots, g_{5}\right)^{\mathrm{T}}
$$

where all $g_{i}$ are zero, except

$$
g_{2}(z)=\theta^{2} u
$$

Note that for all $r>0$, the operator $g(\cdot)$ is Lipschitz in $z$ in the ball $B(0, r)$.

The domain $D(A)$ of the operator $A$ is defined as

$$
\begin{aligned}
D(A)=\{ & \left(u u_{t} \phi \phi, \theta\right)^{\mathrm{T}} \mid u \in \mathbf{H}_{0}^{2}, \phi \in \mathbf{H}_{0}^{2}, u_{t} \in \mathbf{H}_{0}^{1}, \phi_{t} \in \mathbf{H}_{0}^{1}, \theta \in \mathbb{R} \\
& \text { GA } \left.\left[u_{x}(L, t)-\phi(L, t)\right]+\alpha u_{t}(L, t)=0, \operatorname{EI} \phi_{x}(L, t)+\beta \phi_{t}(L, t)=0\right\}
\end{aligned}
$$

In $\mathscr{H}$ we define the following inner-product:

$$
\begin{aligned}
\langle z, \underline{z}\rangle= & \frac{1}{2} I_{\mathrm{R}} \partial \underline{\theta}+\frac{1}{2} \int_{0}^{L} \rho\left[u_{t}+\dot{\theta}(b+x)\right]\left[\underline{u}_{t}+\underline{\theta}(b+x)\right] d x+\frac{1}{2} \int_{0}^{L} \operatorname{EI} \phi_{x} \underline{\phi}_{x} d x \\
& +\frac{1}{2} \int_{0}^{L} I_{\mathbf{B}}\left(\phi_{t}+\dot{\theta}\right)\left(\underline{\phi}_{t}+\underline{\theta}\right) d x+\frac{1}{2} \int_{0}^{L} \mathrm{GA}\left(u_{x}-\phi\right)\left(\underline{u}_{x}-\underline{\phi}\right) d x
\end{aligned}
$$

where $z=\left(u u_{t} \phi \phi, \hat{\theta}\right)^{\mathrm{T}} \in \mathscr{H}$, and $\underline{z}=(\underline{u} \underline{u}, \phi \underline{\phi}, \underline{\hat{\theta}})^{\mathrm{T}} \in \mathscr{H}$.

Note the standard Sobolev norm which makes $\mathscr{H}$ a Banach space is

$$
\|z\|_{1}^{2}=\int_{0}^{L} u^{2} d x+\int_{0}^{L} u_{x}^{2} d x+\int_{0}^{L} u_{1}^{2} d x+\int_{0}^{L} \phi^{2} d x+\int_{0}^{L} \phi_{x}^{2} d x+\theta^{2}
$$

but, by using inequalities (61), (62), (78) and (79), it can be shown that the norm induced by (112) is equivalent to the norm defined by (113) (see Morgül 1989).

\section{Theorem 2}

Consider the linear unbounded operator $A: \mathscr{H} \rightarrow \mathscr{H}$ given by (109). Then

(a) $A$ generates a $C_{0}$ semigroup $T(t)$;

(b) There exist positive contants $M>0$ and $\delta>0$ such that the following holds:

$$
\|T(t)\| \leqslant M \exp (-\delta t) \quad t \geqslant 0
$$

where the norm is that induced by the inner-product defined in (112) (for terminology in semigroup theory, the reader is referred to Pazy 1983).

Proof

(a) We use Lumer-Phillips theorem to prove the assertion (a) (see Pazy 1983). Hence, one has to prove that $A$ is dissipative and that the operator $\lambda I-A: \mathscr{H} \rightarrow \mathscr{H}$ is onto for some $\lambda>0$. 
As before, differentiating the norm induced by (112) we obtain, for all $z \in \mathscr{H}$ :

$$
\frac{d}{d t}\langle z, z\rangle=2\langle z, A z\rangle=-k \theta^{2}-\alpha u_{\imath}^{2}(L, t)-\beta \phi_{\imath}^{2} \leqslant 0
$$

which is the energy estimate (56). This proves that $A$ is dissipative.

To prove that the linear operator $\lambda I-A: \mathscr{H} \rightarrow \mathscr{H}$ for some $\lambda>0$, we decompose the operators $A$ as follows:

$$
A=A_{1}+T_{\mathrm{D}}
$$

where $A_{1}: \mathscr{H} \rightarrow \mathscr{H}$ a linear operator defined as

$$
A_{1}:=\left\{n_{i j}: i, j, \ldots, 5\right\}
$$

were all $n_{i j}$ are zero except

$$
\begin{gathered}
n_{12}=n_{34}=1 \quad n_{25}=k(b+x) \quad n_{45}=k \quad n_{55}=-k \\
n_{21}=\frac{\mathrm{GA}}{I_{\mathrm{R}}} \frac{\partial^{2}}{\partial x^{2}} \quad n_{23}=-\frac{\mathrm{GA}}{\rho} \frac{\partial}{\partial x} \quad n_{41}=\frac{\mathrm{GA}}{I_{\mathrm{B}}} \frac{\partial}{\partial x} \quad n_{43}=\frac{\mathrm{EI}}{I_{\mathrm{R}}} \frac{\partial^{2}}{\partial x^{2}}
\end{gathered}
$$

The operator $A_{1}: \mathscr{H} \rightarrow \mathscr{H}$ is a linear unbounded operator whose domain $D\left(A_{1}\right)$ is equal to $D(A)$ defined by (111). It is known that $A_{1}$ generates a $C_{0}$ semigroup in $\mathscr{H}$ (see Kim 1987). Hence $\lambda I-A: \mathscr{H} \rightarrow \mathscr{H}$ is an invertible operator for all $\lambda>0$.

The operator $T_{\mathrm{D}}: \mathscr{H} \rightarrow \mathscr{H}$ is a degenerate linear operator relative to $A_{1}$ (see Kato 1980). By definition, the range space of $T_{\mathrm{D}}$ is finite dimensional and there exist positive constants $a$ and $b$ such that the following holds:

$$
\left\|T_{\mathrm{D}} z\right\| \leqslant a\|z\|+b\left\|A_{1} z\right\| \quad z \in D\left(A_{1}\right)
$$

That the operator $T_{\mathrm{D}}$ has a finite dimensional range follows from (109), (116) and (117). By using (112), it can be shown that (118) holds for some positive $a$ and $b$. Also, by using the dissipativeness of the operator $A$, it can be proven that for any $\lambda>0$, the operator $I-T_{\mathrm{D}}\left(\lambda I-A_{1}\right)^{-1}: \mathscr{H} \rightarrow \mathscr{H}$ is an invertible linear operator and we have the following:

$$
(\lambda I-A)^{-1}=\left(\lambda I-A_{1}\right)^{-1}\left(I-T_{\mathrm{D}}\left(\lambda I-A_{1}\right)^{-1}\right)^{-1}
$$

which proves that $(\lambda I-A): \mathscr{H} \rightarrow \mathscr{H}$ is onto for all $\lambda>0$ (see Morgül 1989).

This, together with the fact that $A$ is dissipative proves that $A$ generates a $C_{0}$ semigroup in $\mathscr{H}$.

(b) To prove the exponential decay of the semigroup generated by the operator $A$, we first define the energy $E_{1}(t)$ associated with the inner-product (112), that is

$$
E_{1}(t)=\frac{1}{2}\langle z(t), z(t)\rangle
$$

Similar to (57), we define the following function $V_{1}(t)$ :

$$
\begin{aligned}
V_{1}(t)= & 2(1-\varepsilon) t E_{1}(t)+2 \int_{0}^{L} \rho x\left(u_{t}+\theta(b+x)\right) u_{x} d x+2 \int_{0}^{L} I_{\mathrm{B}} x \phi_{x}\left(\phi_{t}+\theta\right) d x \\
& +\delta \int_{0}^{L} I_{\mathrm{B}} \phi\left(\phi_{t}+\theta\right) d x-\delta \int_{0}^{L} \rho u\left[u_{t}+\theta(b+x)\right] d x
\end{aligned}
$$


Following exactly the same procedure as that for the proof of Theorem 1, we obtain the result that $E_{1}(t)$ decays as $O(1 / t)$ for large $t$ (see (60)). Then, exponential decay follows from Pazy's theorem (Pazy 1983, p. 116).

Next we prove the exponential decay of the solutions of (108).

\section{Theorem 3}

Consider (108). Let $T(t)$ be the $C_{0}$ semigroup generated by the linear operator A. Then

(a) for all $z_{0} \in D(A)$, (108) has a unique solution $z(t)$;

(b) in terms of the semigroup $T(t)$ generated by $A$, this classical solution can be written as

$$
z(t)=T(t) z_{0}+\int_{0}^{t} T(t-s) g(z(s)) d s
$$

(c) this classical solution $z(t)$ decays exponentially to zero.

\section{Proof}

(a) Since $A$ generates a $C_{0}$ semigroup $T(t)$ and $g(\cdot): \mathscr{H} \rightarrow \mathscr{H}$ is a $C^{\infty}$ function (see (110)), it follows that for all $z_{0} \in D(A)$, (108) has a unique classical solution defined locally in time. But since for sufficiently large $t, T(t)=O(1 /$ $t$ ) by Theorem 1, it follows that the solution is in fact defined for all $t \geqslant 0$ (see Pazy 1983).

(b) It is well-known that (120) gives the mild solution of (108). But, since the classical solution of (108) exists and is unique, it follows that this mild solution is also a classical solution (see Pazy 1983).

(c) From (110) and (112) it follows that

$$
\|g(z)\|^{2}=\frac{1}{2} \int_{0}^{L} \rho \theta^{4} u^{2} d x \leqslant \frac{1}{2} \rho \theta^{4} M_{1}\|z\|^{2}
$$

where

$$
M_{1}=2 \frac{\max \left\{2 L^{2}, 2 L^{4}\right\}}{\min \left\{I_{\mathrm{R}}, 1, \mathrm{GA}, \mathrm{EI}\right\}}
$$

(see (80)). Since $\theta$ decays at least as $O(1 / t)$, applying the Bellman-Gronwall lemma to (120) and using (114) we conclude that the solution of (108) decays exponentially to zero (see Morgül 1989 a).

\section{Numerical results}

For illustration, we present the results of a numerical simulation of the flexible spacecraft dynamics given by (99)-(104).

For the purpose of simulation, we use finite difference technique with only $N$-point spatial discretization, approximating the spatial partial derivatives by using a central difference formula (see Greenspan and Casulli 1988). Therefore, we obtain $4 N+1$ coupled non-linear ordinary differential equations which we call system $\mathcal{N}$. To simulate this set of equations, we use a trapezoidal integration algorithm. 
First, to show the effect of the controls on the system, we linearize the system $\mathcal{N}$, and find the eigenvalues of the resulting linear system of equations. For this simulation, we choose the following parameters, which were taken for Kim and Renardy (1987): $\rho=1 \mathrm{~kg} \mathrm{~m}^{-1}, \mathrm{GA}=2.8 \times 10^{8} \mathrm{~kg} \mathrm{~m} \mathrm{~s}^{-2}, \mathrm{EI}=6.3 \times 10^{5} \mathrm{~kg} \mathrm{~m}^{3} \mathrm{~s}^{-2}$, $I_{\mathbf{B}}=0.033 \mathrm{~kg} \mathrm{~m}, I_{\mathrm{R}}=100 \mathrm{~kg} \mathrm{~m}^{2}, L=0.1 \mathrm{~m}, b=1 \mathrm{~m}$.

To compute the eigenvalues, we choose the control values as follows:

$$
\begin{aligned}
& \text { case }(i): k=0, \alpha=0, \beta=0, \\
& \text { case (ii): } k=1, \alpha=1, \beta=1, \\
& \text { case (iii): } k=10, \alpha=5, \beta=5, \\
& \text { case (iv): } k=100, \alpha=50, \beta=50 .
\end{aligned}
$$

The first 21 eivenvalues of the linearized equations are shown in Tables 1-4. Note that in case (i) the control parameters are set to zero, hence the resulting system is expected to be conservative (see (56)). This property is reflected in the system eigenvalues as shown in the Table 1 , since all eigenvalues are on the imaginary axis. The eigenvalue at the origin is the one associated with the rigid body motion. In cases (ii) -(iv), we gradually increase the control parameters; as a result, the eigenvalues are shifted to the left hand side of the complex plane. The results are given in Tables $2-4$, in which negative real eigenvalues are associated with rigid body motion.

Tables 2-4 suggest that the eigenvalue associated with rigid motion and the eigenvalues associated with the flexible beam are separated. Hence, a singular perturbation approach may be employed to separate rigid body and flexible beam motion. This point needs further investigation.

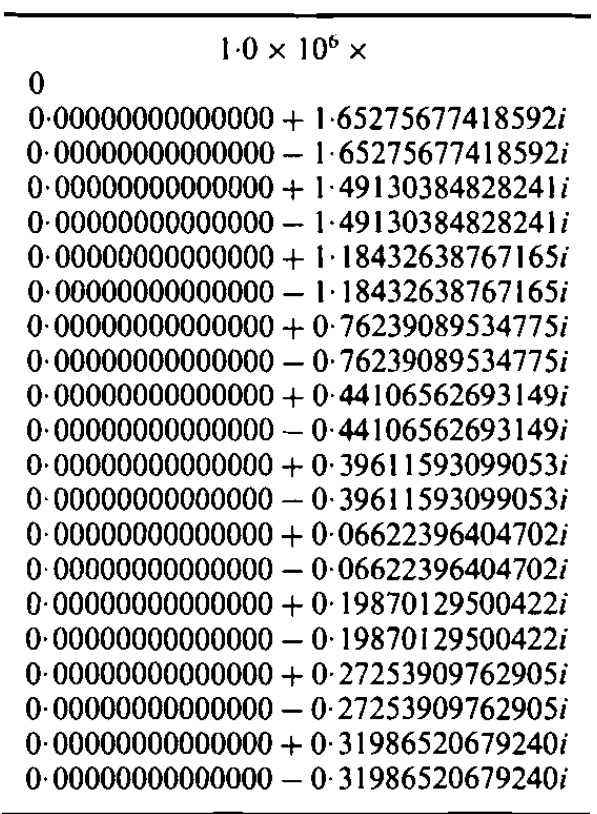

Table 1. Eigenvalues for case (i).

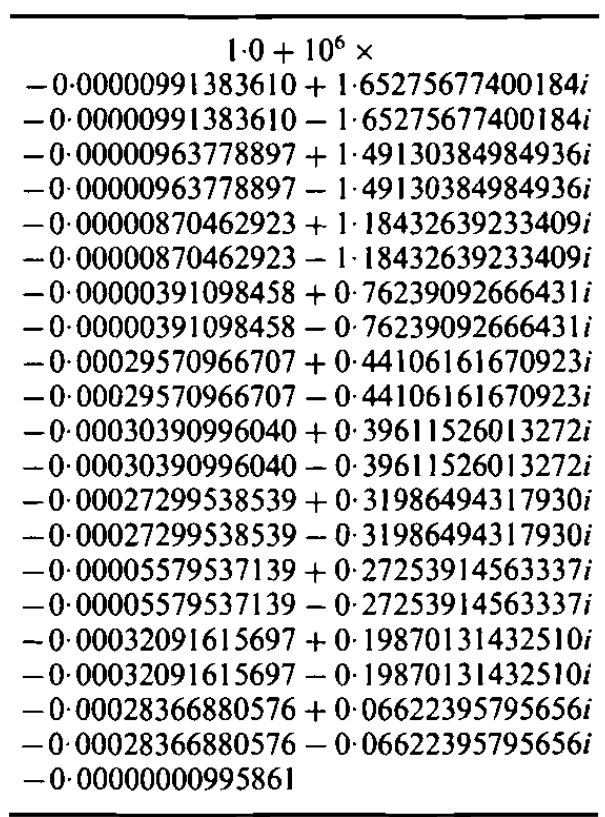

Table 2. Eigenvalues for case (ii). 


\begin{tabular}{|c|}
\hline 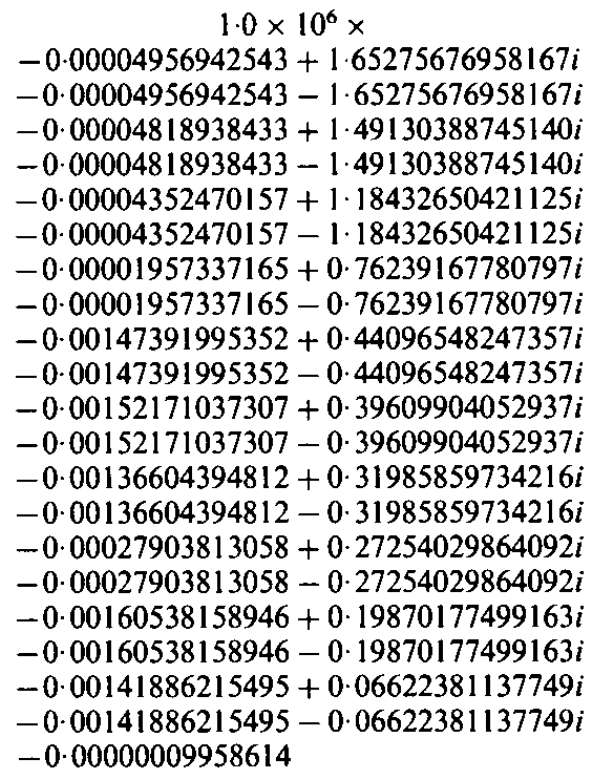 \\
\hline
\end{tabular}

Table 3. Eigenvalues for case (iii).

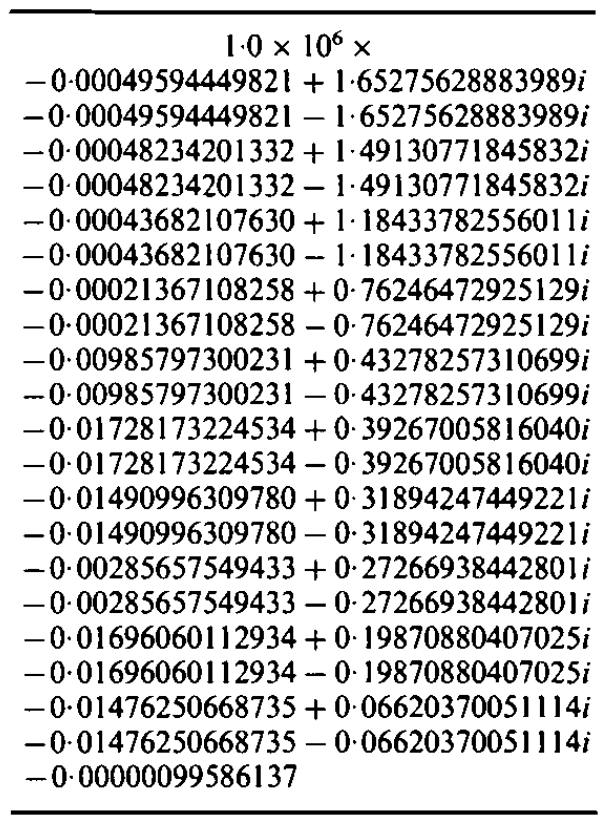

Table 4. Eigenvalues for the case (iv).

For the purpose of illustration, we simulate the equations of the system $\mathcal{N}$ for the following two sets of parameters.

\section{Set 1}

$\rho=1 \mathrm{~kg} \mathrm{~m}^{-1}, \quad \mathrm{GA}=693 \mathrm{~kg} \mathrm{~m} \mathrm{~s}^{-2}, \quad \mathrm{EI}=223 \mathrm{~kg} \mathrm{~m}^{3} \mathrm{~s}^{-2}, \quad I_{\mathrm{B}}=0.033 \mathrm{~kg} \mathrm{~m}$, $I_{\mathrm{R}}=100 \mathrm{~kg} \mathrm{~m}^{2}, L=2 \mathrm{~m}, b=1 \mathrm{~m}$ :

case 1: $k=0, \alpha=0, \beta=0$;

case 2 : $k=1, \alpha=1, \beta=1$;

\section{Set 2}

$\rho=1 \mathrm{~kg} \mathrm{~m}^{-1}, \mathrm{GA}=1.5 \mathrm{~kg} \mathrm{~m} \mathrm{~s}^{-2}, \mathrm{EI}=7.5 \mathrm{~kg} \mathrm{~m}^{3} \mathrm{~s}^{-2}, I_{\mathbf{B}}=1 \mathrm{~kg} \mathrm{~m}, I_{\mathrm{R}}=1 \mathrm{~kg} \mathrm{~m}^{2}$, $L=0.1 \mathrm{~m}, b=1 \mathrm{~m}$ :

case 3: $k=0, \alpha=0, \beta=0$;

case 4: $k=1, \alpha=1, \beta=1$.

Figures 2-7 show the simulation results for the rigid body angular velocity $\omega$, the end point deflection $u(L, t)$ and the end point deflection velocity $u_{t}(L, t)$ in cases 1-4 indicated above. These results show that, in the absence of controls (i.e. cases (1) and (3)), the beam oscillations do not decay, thus making the flexible spacecraft considered here unsuitable for most applications. However, with the application of appropriate control laws (i.e. cases (2) and (4)), these oscillations decay exponentially to zero. 


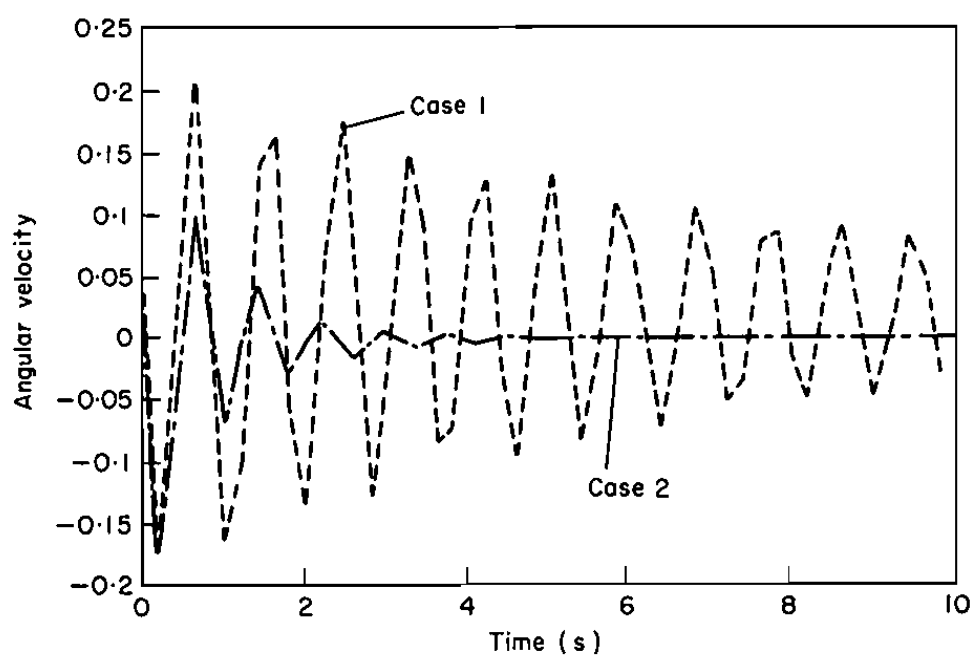

Figure 2. Angular velocity.

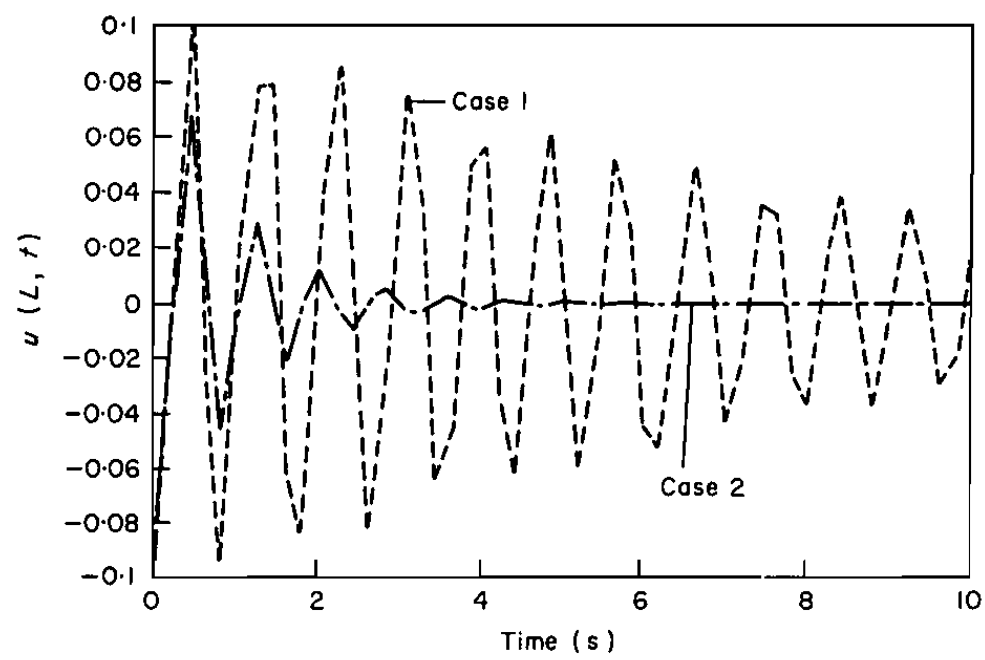

Figure 3. End point displacement.

\section{Conclusion}

In this paper, we have considered the motion of a flexible beam clamped to a rigid body at one end and free at the other. We have assumed that the centre of mass of the rigid body is fixed in an inertial frame and that the mass of the beam is much smaller than the mass of the rigid body, hence the centre of mass of the rigid body is approximately the centre of mass of the whole configuration. For this configuration, we have first used the so-called geometrically exact beam model for the flexible beam to obtain the equations of motion. We then proposed a feedback control law and showed that, together with this control law, the solutions of the 


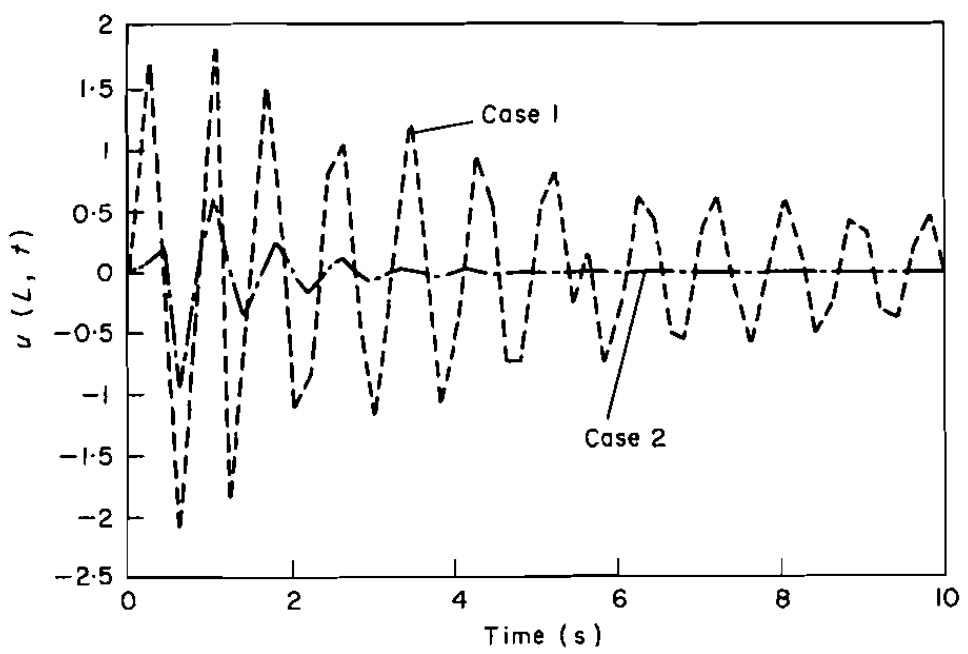

Figure 4. End point velocity.

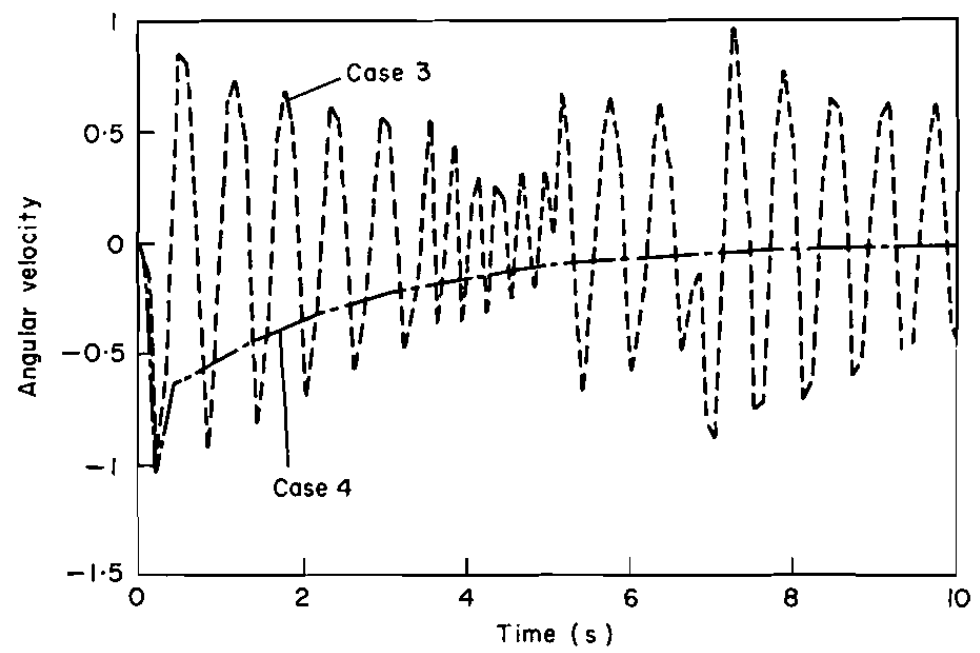

Figure 5. Angular velocity.

equations of motion are stabilized in the sense that the 'energy' of the configuration becomes a non-increasing function of time.

Under the assumption of planar motion linearization of the geometrically exact beam model yields the well-known Timoshenko beam model. By using this linearized model for the beam, we then showed that with the proposed control law, the 'energy' of the configuration, decays exponentially to zero.

Whether we can obtain exponential stabilization by using the geometrically exact beam model without any linearization needs further investigation. Also, application of the boundary control techniques presented in this paper to various problems arising in the control of flexible structures, such as tracking, orientation, etc., could prove useful. 


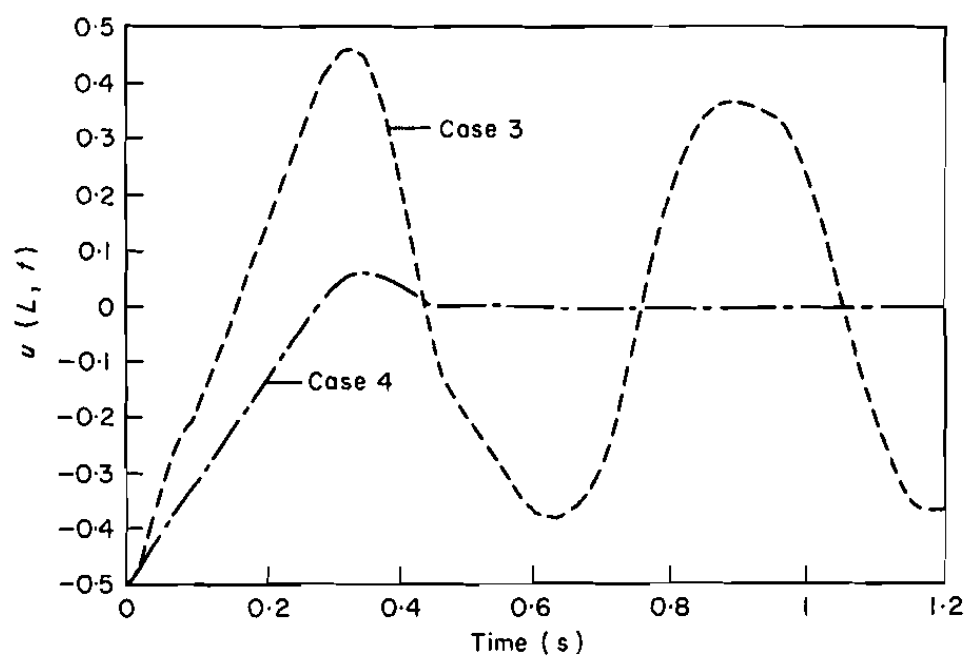

Figure 6. End point displacement.

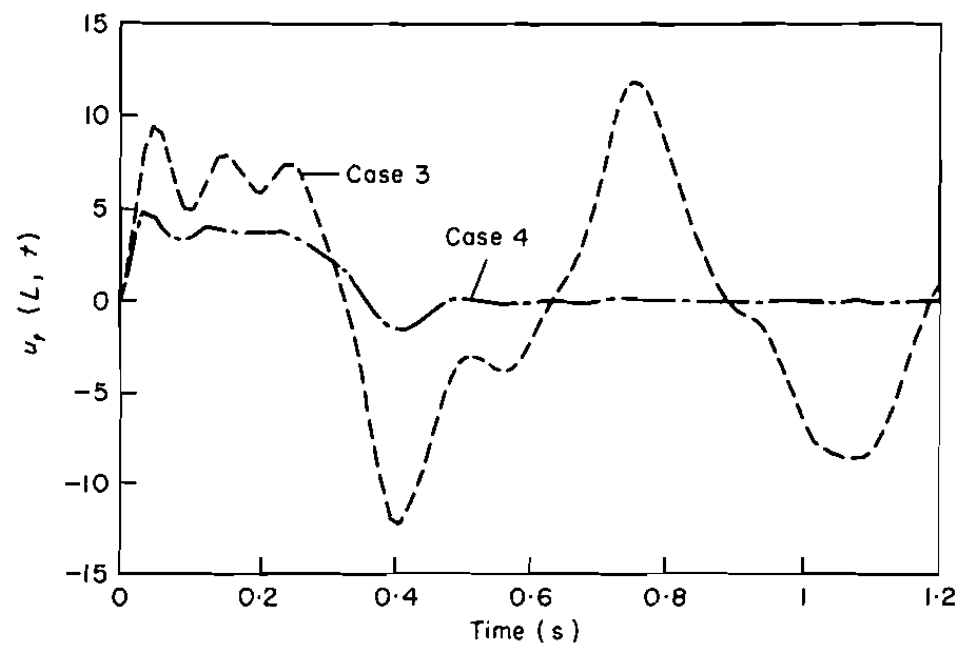

Figure 7. End point velocity.

\section{ACKNOWLEDGMENTS}

The author would like to thank Professor Charles A. Desoer for his guidance, encouragement and careful review of this manuscript, and John Anagnost for many helpful discussions and numerous helpful comments. The author also gratefully acknowledges the help of Ogan Ocal1 for the simulation results presented in the manuscript.

This research has been partially supported by National Science Foundation Grant ECS 8500993 and by the Scientific and Technical Research Council of Turkey. 


\section{REFERENCES}

Antman, S. S., 1972, The theory of rods. Handbuch der Physik, Vol. 6 a/2 (Springer-Verlag). BAKR, E. M., and Shabana, A. A., 1987, Timoshenko beams and flexible multibody system dynamics. Journal of Sound and Vibration, 116, 89-107.

BALAS, M. J., 1978, Modal control of certain flexible dynamical systems. SIAM Journal on Control and Optimization, 16, 450-462; 1982, Trends in large space structure control theory: fondest hopes, wildest dreams. I.E.E.E. Transactions on Automatic Control, 27, 522-535.

Biswas, S. K., and AHMED, N. U., 1986, Stabilization of a class of hybrid systems arising in flexible spacecraft. Journal of Optimization Theory and Applications, 50, 83-108.

CHEN, G., 1979, Energy decay estimates and exact boundary value controllability for the wave equation in a bounded domain. Journal de Mathématique Pures et Applicées, 58, 249-273.

Chen, G., Delfour, M., Krall, A. M., and Payre, G., 1987 a, Modeling, stabilization and control of serially connected beams. SIAM Journal on Control and Optimization, 25, 526-546.

Chen, G., Krantz, S. G., Ma, D. W., Wayne, C. E., and West, H. H., 1987 b, The Euler-Bernoulli beam equation with boundary energy dissipation. Operator methods for optimal control problems. Edited by S. J. Lee, Lecture Notes in Pure and Applied Mathematics Series (New York: Marcell-Dekker Inc) 67-96.

Delfour, M. C., Lagnese, J., and Polis, M. P., 1986, Stabilization of hyperbolic systems using concentrated sensors and actuators. I.E.E.E. Transactions on Automatic Control, 31, 1091- 1096

Desoer, C. A., and Morgül, Ö., 1988, Control and stabilization of a flexible beam attached to a rigid body: planar motion. Proceedings of the 27th Conference on Decision and Control, Austin, Texas pp. 1643-1645.

Goldstein, H., 1980, Classical Mechanics, second edition (Reading, Mass: AddisonWesley).

Greenspan, D., and Casull, V. 1988, Numerical Analysis for Applied Mathematics, Science and Engineering (Reading, Mass: Addison-Wesley).

KATO, T., 1980, Perturbation Theory for Linear Operators, second edition (New York: Springer-Verlag)

KIM, J. U., and RENARDY, Y., 1987, Boundary control of the Timoshenko beam. SIAM Journal on Control and Optimization, 25, 1417-1429.

Marsden, J. E., and Hughes, T. J. R., 1983, Mathematical Foundations of Elasticity (Englewood Cliffs, NJ: Prentice-Hall), p. 4

Meirovitch, L., 1967, Analytical Methods in Vibration (New York: MacMillan), pp. $129-132$.

Morgül, Ö., 1989, Problems in the control of flexible spacecraft, Ph.D. thesis, Technical Memorandum, Electronics Research Laboratory, Memorandum No. UCB/ERL M89/ 97, University of California, Berkeley; 1990, Control and stabilization of a flexible beam attached to a rigid body. International Journal of Control., 11-33.

PAZY, A., 1983, Semigroups of Linear Operators and Applications to Partial Differential Equations (New York: Springer-Verlag), pp. 183-189.

QuinN, J. P., and Russel, D. L., 1978, Asymptotic stability and energy decay rates for solutions of hyperbolic equations with boundary damping. Proceedings of the Royal Society of Edinburgh, Section A, (77), 1977/1978, 97-127.

Royden, H. L., 1968, Real Analysis, second edition (New York: MacMillan) p. 110.

RUSSELL, D. L., 1986, Mathematical models for the elastic beam and their control-theoretic implications. Semigroups, Theory and Applications, Vol. 2, Edited by H. Brezis, M. G. Crandall and F. Kappel, (Essex, UK: Longman Scientific and Technical), pp. $177-217$.

Simo, J. C., 1985, A finite strain beam formulation: a three dimensional dynamic problem. Part 1. Computational Methods in Applied Mechanical Engineering, 49, 55-70.

VU-QuoC, L., 1986, Dynamics of flexible structures performing large overall motions: a geometrically nonlinear approach. Ph.D. dissertation, College of Engineering, University of California, Berkeley. 